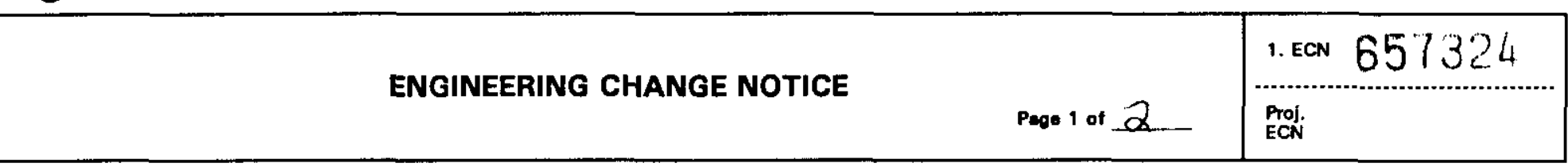

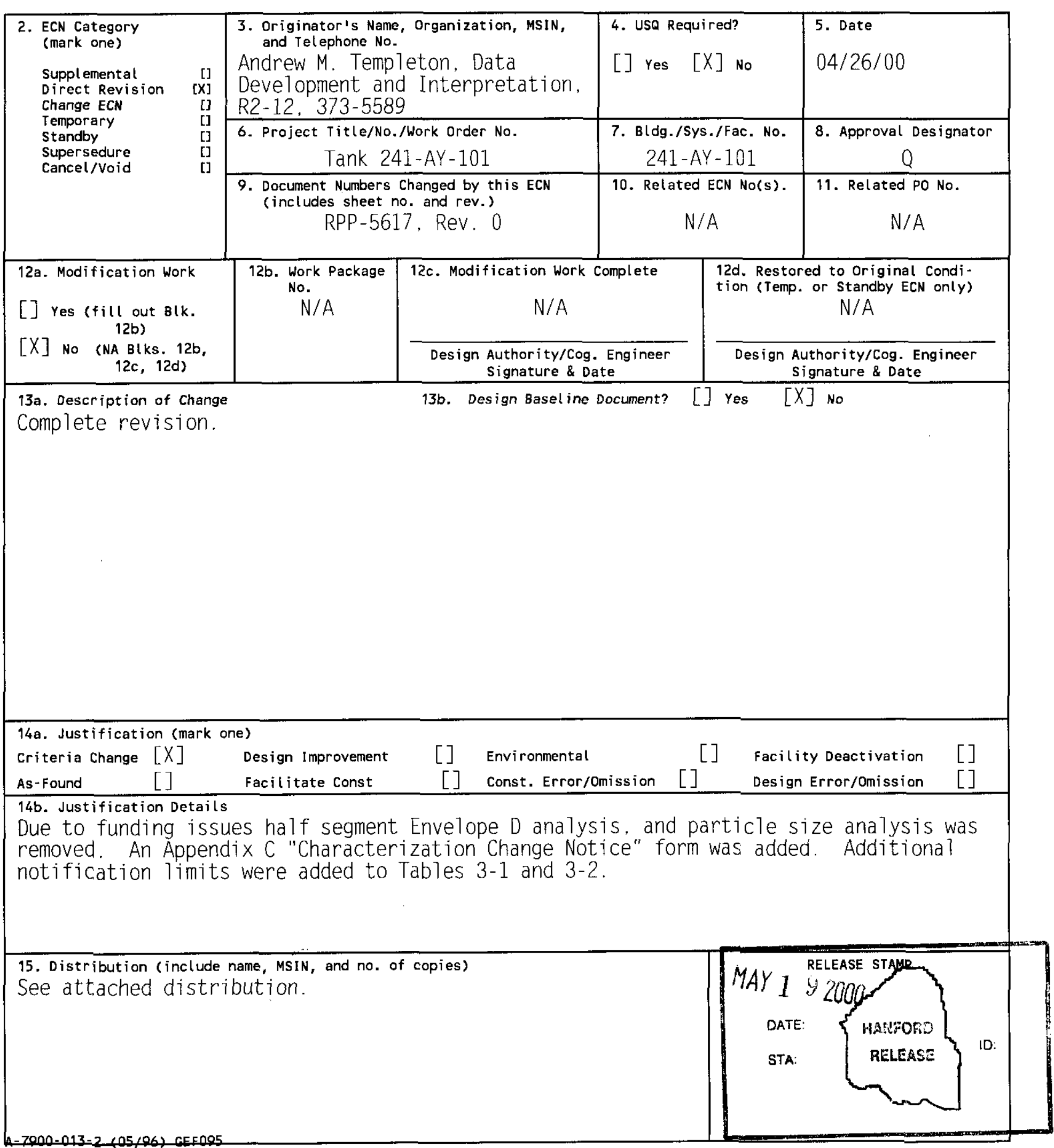




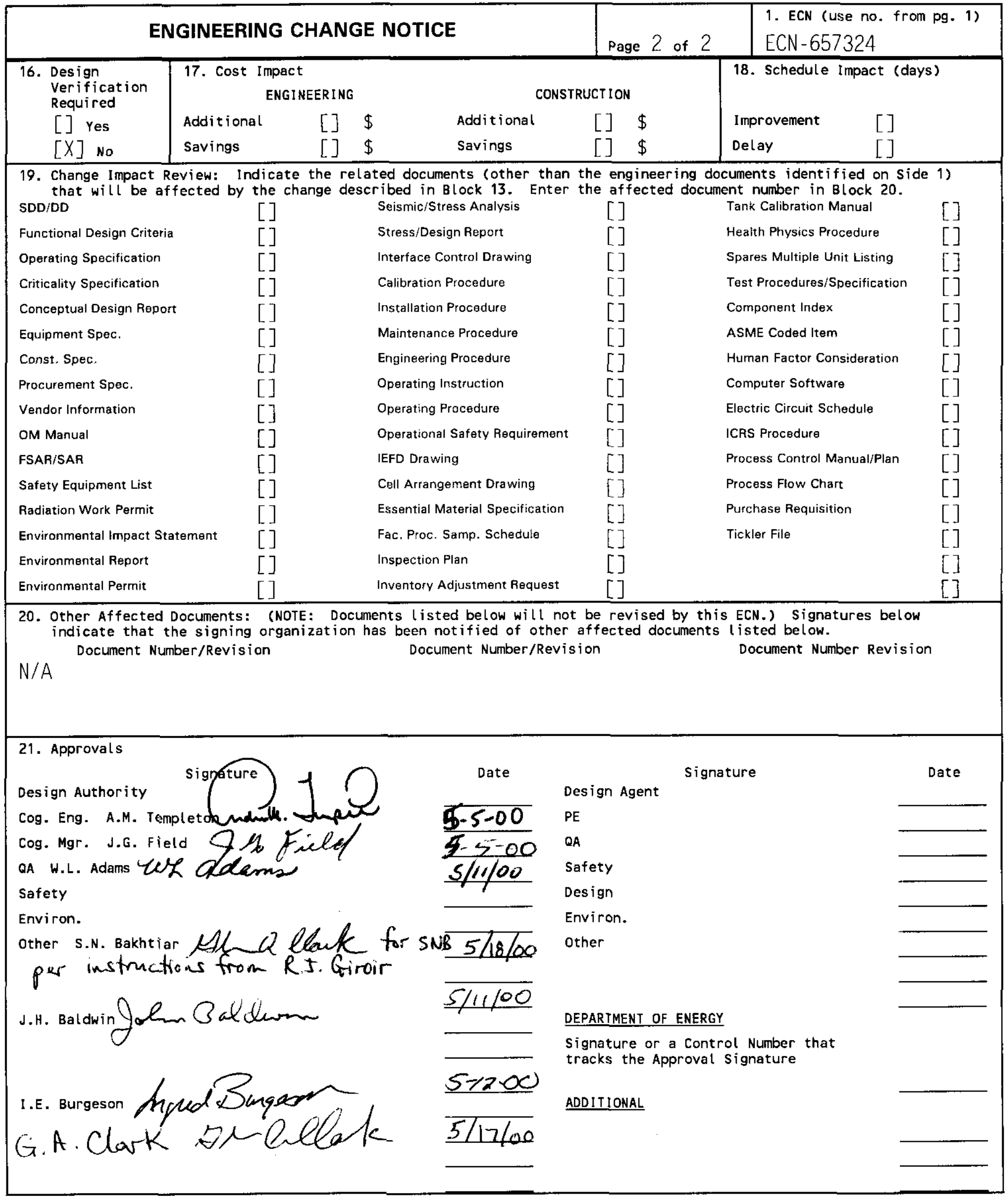




\section{Tank 241-AY-101 Privatization Push Mode Core Sampling and Analysis Plan}

Andrew M. Templeton

CH2M HILL Hanford Group. Inc., Richland, WA 99352

U.S. Department of Energy Contract DE-AC06-96RL13200

EDT/ECN: $\quad$ ECN-657324

Org Code: $74 \mathrm{~B} 20$

B\&R Code: EW 3120074 Total Pages: 8

Key Words: Tank 241-AY-101, 241-AY-101, Tank AY-101, AY-101, AY Farm, Privatization, Push Mode, Push, Core, Sampling, Analysis

Abstract: N/A

TRADEMARK DISCLAIMER. Reference herein to any specific commercial product, process, or service by trade name, trademark, manufacturer, or otherwise, does not necessarily constitute or imply its endorsement, recormendation, or favoring by the United States Government or any agency thereof or its contractors or subcontractors.

Printed in the United States of America. To obtain copies of this document, contact: Document Control Services, P.O. Box 950, Mailstop H6-08, Richland WA 99352, Phone (509) 372-2420; Fax (509) 376-4989.
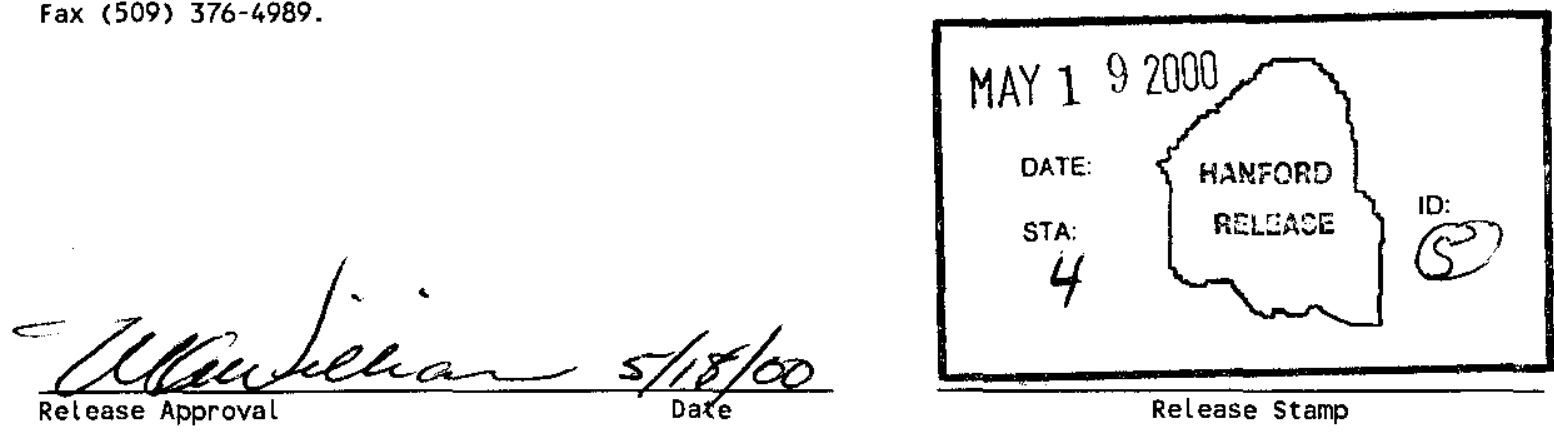

\section{Approved for Public Release}




\section{RECORD OF REVISION}

(1) Document Number

RPP -5617

Page 1

(2) Title

Tank 241-AY-101 Privatization Push Mode Core Sampling and Analysis Plan

CHANGE CONTROL RECORD

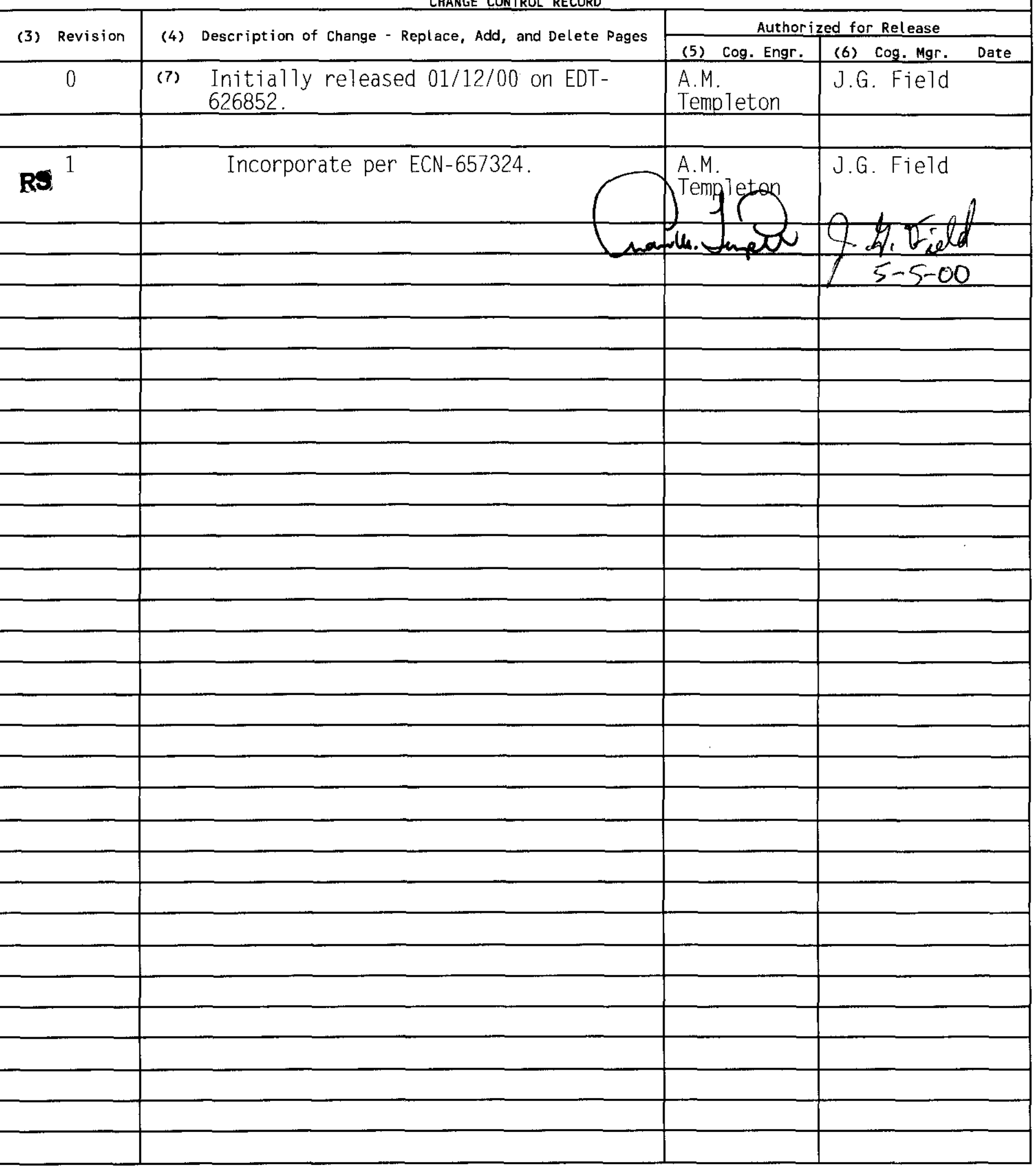


RPP-5617

Revision 1

\title{
Tank 241-AY-101 \\ Push Mode Core Sampling and Analysis Plan
}

\author{
A. M. Templeton \\ CH2M HILL Hanford Group, Inc.
}

Date Published

May 2000

Prepared for the U.S. Department of Energy

Office of River Protection 


\section{TABLE OF CONTENTS}

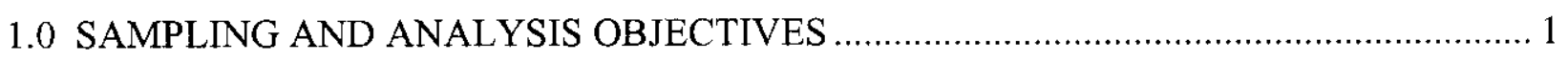

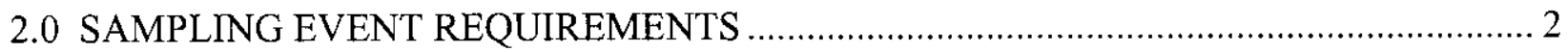

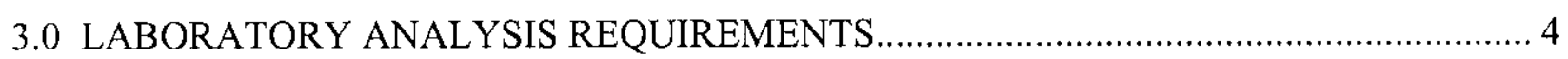

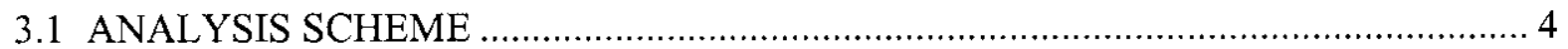

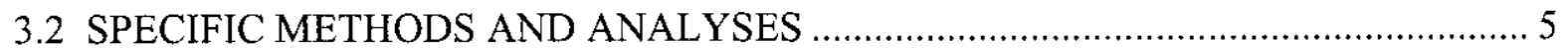

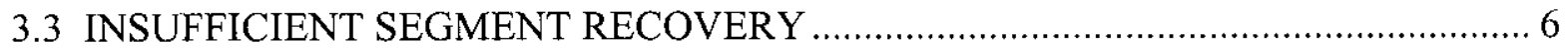

4.0 QUALITY ASSURANCE AND QUALITY CONTROL ................................................. 22

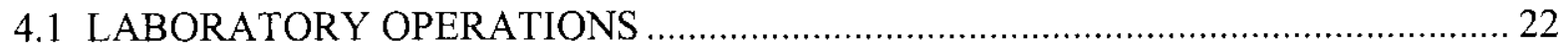

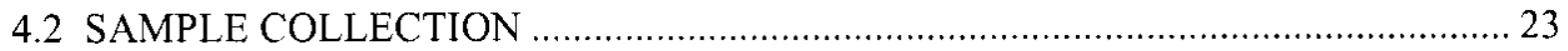

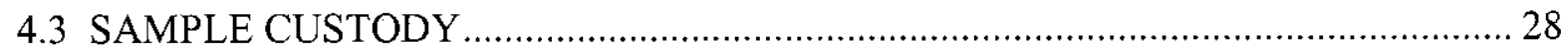

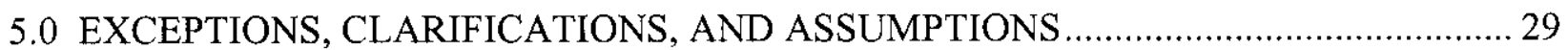

5.1 EXCEPTIONS TO DATA QUALITY OBJECTIVES REQUIREMENTS .................. 29

5.2 CLARIFICATIONS AND ASSUMPTIONS ………................................................ 30

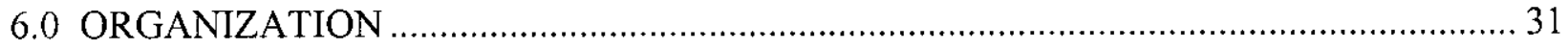

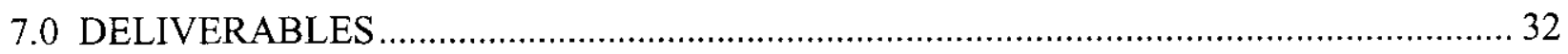

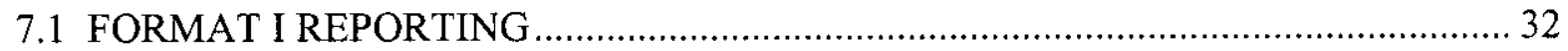

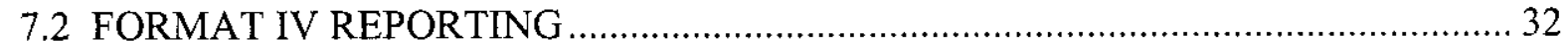

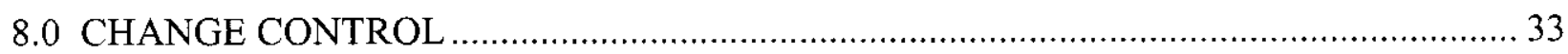

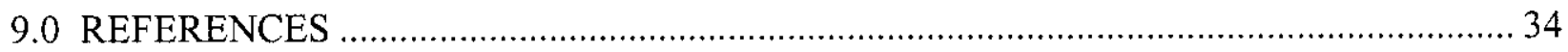

\section{APPENDICES}

APPENDIX A: EXPECTED PHYSICAL PROFILE OF TANK 241-AY-101 CORE SAMPLES

APPENDIX B: 241-AY-101 WASTE COMPOSITE PREPARATION GUIDELINES ............. B-1

APPENDIX C: CHARACTERIZATION CHANGE NOTICE FORM ………………….......... -1 
RPP-5617, Rev. 1

\section{LIST OF TABLES}

Table 3-1. Tank 241-AY-101 Chemical, Radiological, and Physical Analytical Requirements: Liquids.

Table 3-2. Tank 241-AY-101 Chemical, Radiological, and Physical Analytical Requirements:

Solids

Table 3-3. Detection Limits and Minimum Reportable Quantities for Low-Activity Waste Liquids 12

Table 3-4. Detection Limits and Minimum Reportable Quantities for High Level Waste Solids 16

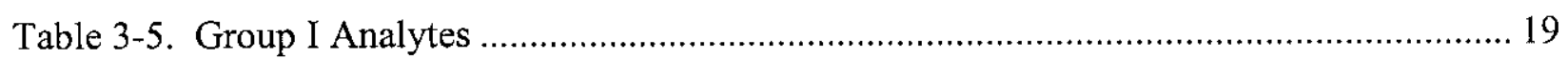

Table 4-1. Quality Control Parameters for Liquid Analysis............................................. 24

Table 4-2: Quality Control Parameters for Solids Analysis ............................................. 26

Table 6-1. Tank 241-AY-101 Project Points of Contact and Key Personnel List..................... 31

Table A-1: Tank 241- AY-101 Physical Profile Estimate Risers 15G and 15O ....................A-2 


\section{LIST OF ABBREVIATIONS}

\begin{tabular}{|c|c|}
\hline AEA & alpha energy analysis \\
\hline AMU & atomic mass unit \\
\hline $\mathrm{CHG}$ & CH2M HILL Hanford Group, Inc. \\
\hline $\mathrm{Ci} / \mathrm{g}$ & curies per gram \\
\hline $\mathrm{cm}$ & centimeters \\
\hline $\mathrm{CPO}$ & Characterization Project Operations \\
\hline CVAA & cold vapor atomic absorption \\
\hline DQO & data quality objective \\
\hline $\mathrm{FH}$ & Fluor Hanford \\
\hline $\mathrm{ft}$ & feet \\
\hline $\mathrm{g}$ & gram \\
\hline GEA & gamma energy analysis \\
\hline GFAA & graphite furnace atomic absorption \\
\hline HLW & high level waste \\
\hline IC & ion chromatography \\
\hline $\mathrm{ICP} / \mathrm{AES}$ & inductively coupled plasma/atomic emission spectroscopy \\
\hline $\mathrm{ICP} / \mathrm{MS}$ & inductively coupled plasma/mass spectrometry \\
\hline ISE & ion-specific electrode \\
\hline kgal & kilogallon \\
\hline $\mathrm{kL}$ & kiloliter \\
\hline $\mathrm{L} \& \mathrm{H}$ & low-activity waste and high-level waste \\
\hline LAW & low activity waste \\
\hline LCS & laboratory control standard \\
\hline LFL & lower flammability limit \\
\hline $\mathrm{LiBr}$ & lithium bromide \\
\hline LMHC & Lockheed Martin Hanford Corporation \\
\hline MRQ & minimum reportable quantity \\
\hline MSL & mean sea level \\
\hline N/A & not applicable \\
\hline NHC & Numatec Hanford Corporation \\
\hline NP & not performed \\
\hline NS & not specified \\
\hline PIC & person in charge \\
\hline PNNL & Pacific Northwest National Laboratory \\
\hline QA & quality assurance \\
\hline QC & quality control \\
\hline RSD & relative standard deviation \\
\hline RPP & River Protection Project \\
\hline SAP & sampling and analysis plan \\
\hline Sep. & separation \\
\hline TBD & to be determined \\
\hline TIC & total inorganic carbon \\
\hline
\end{tabular}




\section{RPP-5617, Rev. 1}

TOC

TWRS

WFD

WIT

WMH

$\mathrm{wt} \%$

$\mu \mathrm{Ci} / \mathrm{g}$

$\mu \mathrm{Ci} / \mathrm{mL}$

$\mu \mathrm{g} / \mathrm{g}$

$\mu \mathrm{g} \mathrm{C} / \mathrm{g}$

$\mu \mathrm{g} / \mathrm{mL}$

$\%$ total organic carbon

Tank Waste Remediation System (now RPP)

Waste Feed Delivery

Waste Integration Team

Waste Management Hanford

weight percent

microcurie per gram

microcuries per milliliter

micrograms per gram

micrograms carbon per gram

micrograms per milliliter

percent 


\subsection{SAMPLING AND ANALYSIS OBJECTIVES}

This sampling and analysis plan (SAP) identifies characterization objectives pertaining to sample collection, laboratory analytical evaluation, and reporting requirements for samples obtained from tank 241-AY-101. The purpose of this sampling event is to obtain information about the characteristics of the contents of 241-AY-101 required to satisfy Data Quality Objectives For RPP Privatization Phase 1: Confirm Tank T Is An Appropriate Feed Source For High-Level Waste Feed Batch X (HLW DQO) (Nguyen 1999a), Data Quality Objectives For TWRS Privatization Phase 1: Confirm Tank T Is An Appropriate Feed Source For Low-Activity Waste Feed Batch X (LAW DQO) (Nguyen 1999b), Low Activity Waste and High-Level Waste Feed Data Quality Objectives (L\&H DQO) (Patello et al. 1999), and Characterization Data Needs for Development, Design, and Operation of Retrieval Equipment Developed through the Data Quality Objective Process (Equipment DQO) (Bloom 1996). Special instructions regarding support to the LAW and HLW DQOs are provided by Baldwin (1999).

Push mode core samples will be obtained from risers $15 \mathrm{G}$ and $15 \mathrm{O}$ to provide sufficient material for the chemical analyses and tests required to satisfy these data quality objectives. The 222-S Laboratory will extrude core samples; composite the liquids and solids; perform chemical analyses on composite and segment samples; archive half-segment samples; and provide subsamples to the Process Chemistry Laboratory. The Process Chemistry Laboratory will prepare test plans and perform process tests to evaluate the behavior of the 241-AY-101 waste undergoing the retrieval and treatment scenarios defined in the applicable DQOs. Requirements for analyses of samples originating in the process tests will be documented in the corresponding test plans and are not within the scope of this SAP. 


\subsection{SAMPLING EVENT REQUIREMENTS}

As of January 11, 1999, surveillance readings indicated that tank 241-AY-101 contained a total waste volume of approximately $571 \mathrm{~kL}(151 \mathrm{kgal})$, consisting of $223 \mathrm{~kL}$ (59 kgal) of supernate and $356 \mathrm{~kL}$ ( $94 \mathrm{kgal}$ ) of sludge. This waste volume is equivalent to $139.7 \mathrm{~cm}$ (55 inches) of waste as measured from the inside bottom of the tank. A physical profile prediction based on waste fill history and previous sampling information is provided in Appendix A. The waste consists primarily of dilute complexed waste.

Tank 241-AY-101 will be push mode core sampled using a push mode core sampling truck. A total of four core samples, consisting of 3 segments each, are expected to be taken from two 6 -inch diameter risers $15 \mathrm{G}$ and 150 (two cores from each riser). Within each 6-inch riser, the cores are to be taken from opposite sides of the riser. A minimum of four hours shall elapse between the sampling the first and second cores from a riser. The sampling objective is to obtain four full vertical profiles of the waste; therefore, additional segments may need to be taken depending on the accuracy of the current waste volume records in comparison to pre-sampling zip cord readings. The bottom two segments of each core shall be $x$-rayed; if sufficient solids are not recovered (at least 62.5 inches of solids from each riser), collection of additional solids may be required.

No flow samplers will be used for these samples. If quality-affecting changes to the sampling requirements must be made (including the risers, sampling truck, or segments to be sampled), the change must be recorded and approved by the cognizant engineer and tank coordinator before sampling. This information may be recorded on a permanent data sheet or recorded directly in sampling work package ES-99-00309 for riser 15G and ES-99-00310 for riser 15O. These work packages will contain the operating procedures and the chain-of-custody records for this sampling event.

Prior to core sampling, the dome space (below the riser) shall be measured for the presence of flammable gases. The measurement shall be taken from within the dome space and the data reported as a percentage of the lower flammability limit (LFL). The results shall be transmitted to the tank coordinator within ten working days of the sampling event (Schreiber 1998). If the results are above 25 percent of the LFL when analyzing by gas chromatography/mass spectrometry or gas-specific monitoring gauges, or above 10 percent of the LFL when analyzing with a combustible gas meter, the necessity for recurring sampling for flammable gas concentration and the frequency of such sampling will be determined by the Flammable Gas Safety Project. Any additional vapor sampling is not within the scope of this SAP.

One field blank for tank 241-AY-101 shall be obtained in accordance with procedure TO-060-003. The Characterization Project Operations (CPO) person in charge (PIC) or the PIC designate will verify that the field blank is properly created and shipped. For sampling events having multiple PICs, CPO shall determine which PIC will be responsible for the field blank. This field blank is to accompany the samples to the laboratory. All collected samples shall be shipped to the laboratory following the Load/Transport Sample Cask(s) procedure (TO-080-090). Core samples should be transported to the laboratory within three calendar days from the time each segment is removed from the tank. 
If lithium bromide ( $\mathrm{LiBr}$ ) solution is used in the collection of the core samples, it should be a $0.3 \pm 0.01$ molar solution with a $\mathrm{pH}$ greater than 8 . Characterization Project Operations must state the batch number (barrel number and preparation date) and amount of fluid added at each segment. This information should be indicated on the chain-of-custody form that accompanies the sample to the laboratory. A sample of the $\mathrm{LiBr}$ solution must be provided to the laboratory. This sample shall consist of a container filled with $\mathrm{LiBr}$ solution from the same batch of $\mathrm{LiBr}$ solution used during the sampling. This solution shall be analyzed for lithium and bromide in order to determine the concentration of the tracer at the time the sample was taken. If analysis of the waste samples indicates contamination by the $\mathrm{LiBr}$ solution, these data will be used to determine the amount of contamination. If more than one batch of $\mathrm{LiBr}$ solution is used during sampling event, one solution sample must be provided for each batch in addition to the field blank. 
RPP-5617, Rev. 1

\subsection{LABORATORY ANALYSIS REQUIREMENTS}

The extrusion, sub-sampling, forming a composite, and analysis requirements are described below. For core samples from tank 241-AY-101, the shipping container must be vented every 27 days to release any accumulated gas.

\subsection{ANALYSIS SCHEME}

Data quality objectives requirements drive the analysis of the samples. In order to comply with the LAW, HLW, L\&H, and the Equipment DQOs, the following steps shall be performed on each sample. It should be noted that while the steps presented are the designated path forward when sufficient material is available, the specific information about which segment material (e.g., primary segment sample or retake material) will be used for each specific analysis, will be provided by the tank coordinator.

Complete homogenization of the solid samples prior to forming the composite, during the preparation of the composite, and while subsampling, for analysis is critical to the L\&H DQO. If it is determined that homogenizing at each stage with a tissue homogenizer is not feasible due to sample mass constraints, stop work and contact the tank coordinator for further directions. A decision will be made on how to proceed by the tank coordinator and the Waste Integration Team (WIT) technical point of contact.

(1) Extrude segments, videotaping the extrusion and photographing the extruded segments. The extrusion procedure is LO-160-103 at the 222-S Laboratory. If the segment contains solids, the solids shall be examined to confirm that layering is not present.

(2) Subsample each solids segment for particle size, before waste is handled further. If observations indicate that layers are present, the cognizant scientist directs subsampling for particle size. Archive the particle size samples.

(3) Divide each solids segment into upper and lower halves and perform shear strength on upper and lower halves. Homogenize each bottom-most half segment and subsample for Envelope D analytes per Table 3-2 and archive. Half segment archiving on the bottom-most segment is required by Baldwin (1999).

(4) Separately homogenize solid segments from cores 276 and 278.

(5) Archive the material from cores 275 and 277 for future blending studies with C-104.

(6) Allow drainable liquid samples to settle for at least 16 hours. Record volume percent solids, and note any evidence of gas releases or separated liquid phases.

(7) Prepare 3 separate composites for each riser as directed by tank coordinator per the guidelines in Appendix B.

(8) Archive one of the composites. 
(9) Composites: Mix solids and liquids and allow to settle for at least 16 hours. Record weight, volume, and volume \% settled solids for each composite, and note any evidence of floating layers (organics or solids) or gas generation.

(10) Submit one of the composites (or $175 \mathrm{~g}$ of solids) from each riser to the Process Chemistry Laboratory for solids dissolution screening tests and subsample the "slurry" for $w t \%$ solids and wt $\%$ oxide prior to solids/liquid separation per L\&H DQO. The subsample may need to be removed while mixing. These tests will be performed per an approved Test Plan to be issued at a later date. Wt $\%$ oxide is also requested on the centrifuge solids from step 1.

(11) Separate the composite from each riser by centrifugation. The responsible chemist shall ensure that the centrifuged solids contain no separate liquid phase.

(12) Analyze 3 subsamples of solid fraction of each riser composite separated in step 13 per Table 3-2.

(13) Analyze 3 subsamples of liquid fraction of each riser composite separated in step 13 per Table 3-1.

If liner liquid is observed during extrusion and the liquid is in sufficient quantity to collect, the liner liquid may be retained and analyzed at the discretion of the tank coordinator. In this event, this addition of analyses may not require a revision to this SAP.

Opportunistic analyses as defined in Kristofzski (1996) are to be included when the laboratory is not operating at maximum capacity. Any decisions, observations, or deviations made to this work plan, or during the sample breakdown and analyses shall be documented in writing with justification. These decisions and observations shall be reported in the data report. The reporting formats for analyses are contained in Tables 3-1 and 3-2 and are described in Section 7.0 .

\section{A material balance needs to be performed to account for all the material sampled and composited.}

\subsection{SPECIFIC METHODS AND ANALYSES}

The analyses in Tables 3-1 and 3-2 to be performed on the tank 241-AY-101 core samples are based on the HLW, LAW, L\&H, and Equipment DQOs. The laboratory procedure numbers which shall be used for the analyses are included in the tables. Sample preparation procedures that may be used at the 222-S Laboratory are LA-549-141 for fusion digestion, LA-505-159 or LA-505-163 for acid digestion of samples, and LA-504-101 for water leach of solids. The laboratory is to notify the tank coordinator once analyses are complete.

Duplication of effort shall be avoided where practical. For example, many of the analyses required for the L\&H DQO are also required by the HLW and LAW DQOs. If process testing per the HLW and LAW DQOs determines that dilution is not required for waste retrieval, 
analyses per the L\&H DQO can meet the corresponding data needs of the HLW and LAW DQOs. However, if process testing determines that dilution of the waste is required, then separate analyses of the diluted samples will be as specified in the test plan.

The HLW, LAW, and L\&H DQOs specify minimum reportable quantities (MRQs) of those analytes listed in Tables 3-1 and 3-2. The laboratory is to notify the tank coordinator of any circumstances which prevent achieving detection limits at or below the MRQ for any analyte, and recommend a corrective course of action. The MRQs are provided in Tables 3-3 and 3-4.

The L\&H DQO requires repreparation and/or reanalysis of a subsample under certain conditions. Repreparation and/or reanalysis of a subsample applies to Group 1 analytes whose relative standard deviation (RSD) exceeds the criteria in Section 4 and whose concentration is greater than the MRQ. In such circumstances, only one additional analysis is required, and the results of all analyses shall be reported. The decision to reanalyze a subsample or reprepare and reanalyze a new subsample shall be made by the project coordinator based on the previous results. If after reanalysis, the RSD of all subsamples exceeds the criteria, a decision will be made on the need for further analysis by the project coordinator and the Waste Integration Team (WIT) technical point of contact. Group 1 analytes, as defined by the L\&H DQO for LAW and HLW are listed in Table 3-5.

\subsection{INSUFFICIENT SEGMENT RECOVERY}

If the amount of material recovered from samples taken from 241-AY-101 is insufficient to form the composites and perform the analyses requested in the SAP, the laboratory shall notify the tank coordinator within one working day. It is estimated that at least $1,125 \mathrm{~g}$ of settled solids (about 62.5 inches of solids) per riser will be required. At that time, a priority of the analyses and/or composite scheme may be provided to the laboratory. Any analyses prescribed by the SAP, but not performed, shall be identified in the appropriate data report with justification provided for nonperformance. 


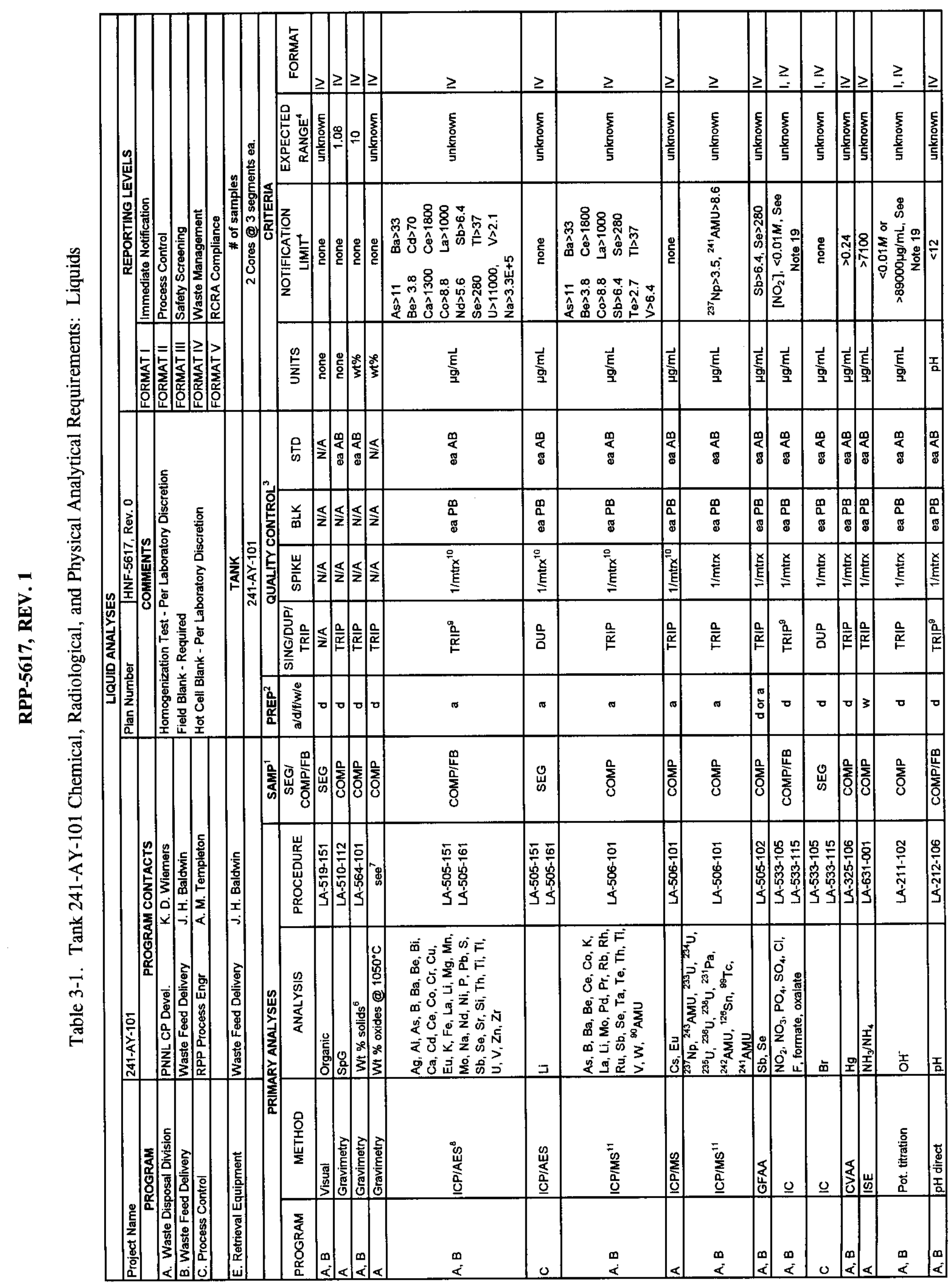




\section{萻}

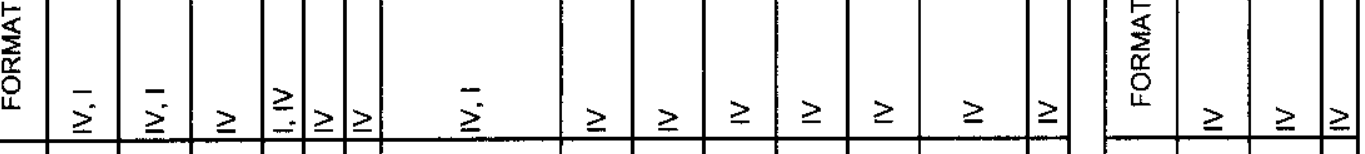

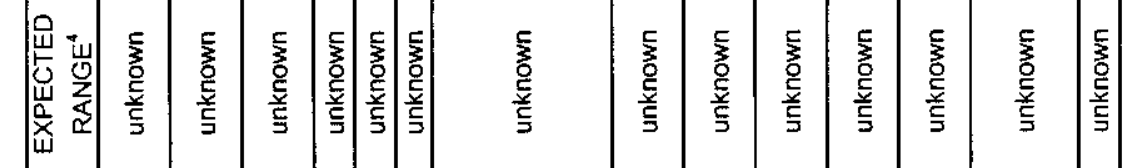

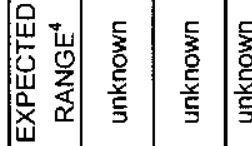

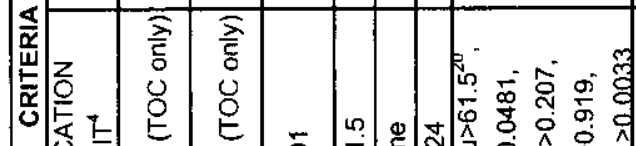

:



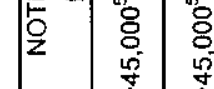

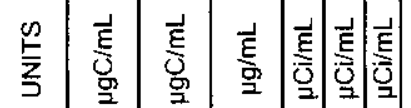

$\underset{\mathrm{E}}{\overrightarrow{\mathrm{O}}}$

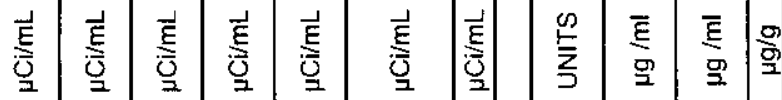

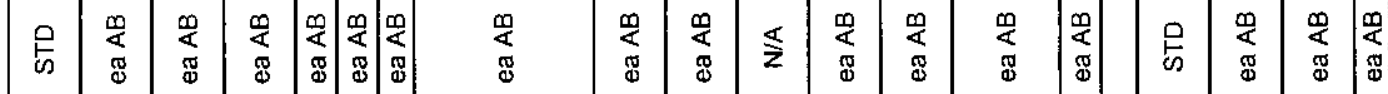

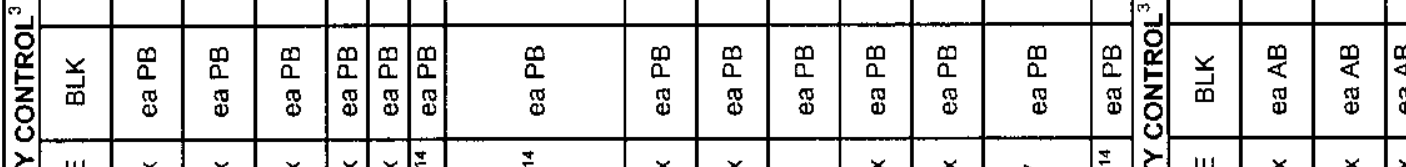

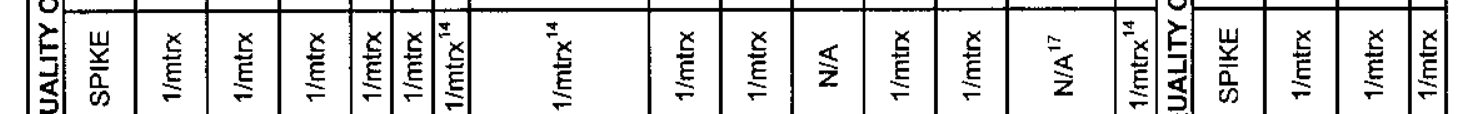

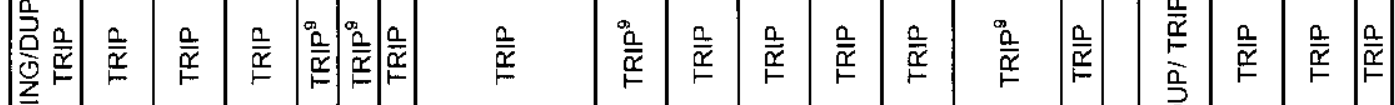

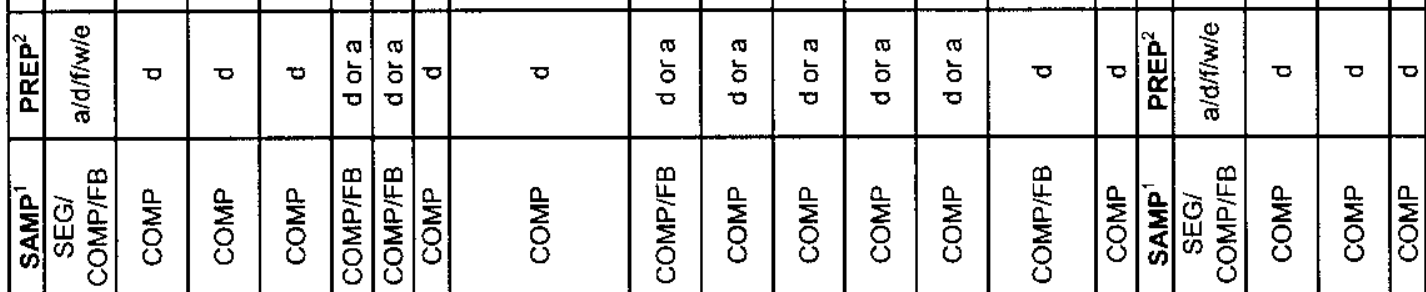

$\Xi$

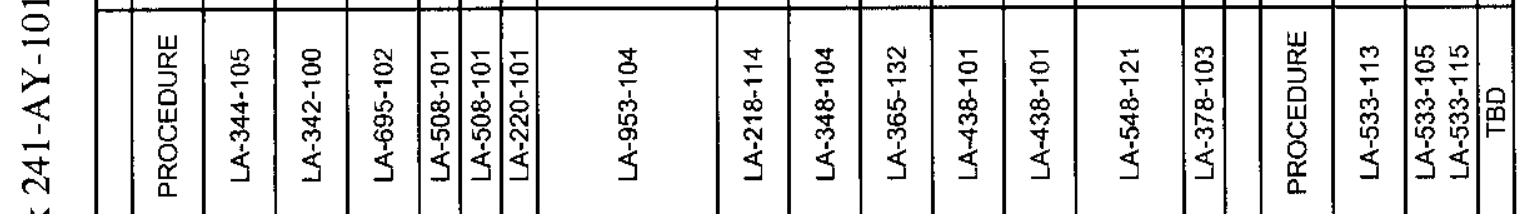

雳

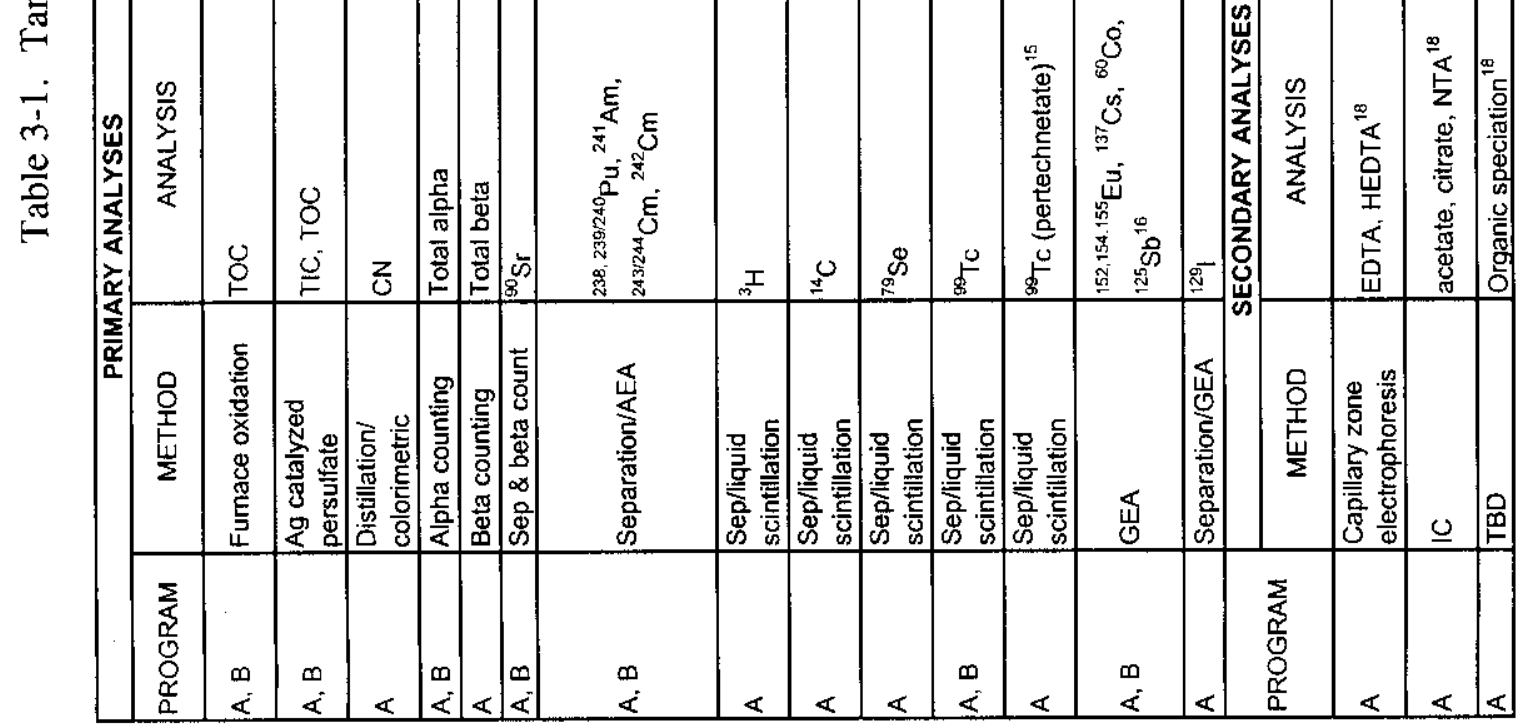

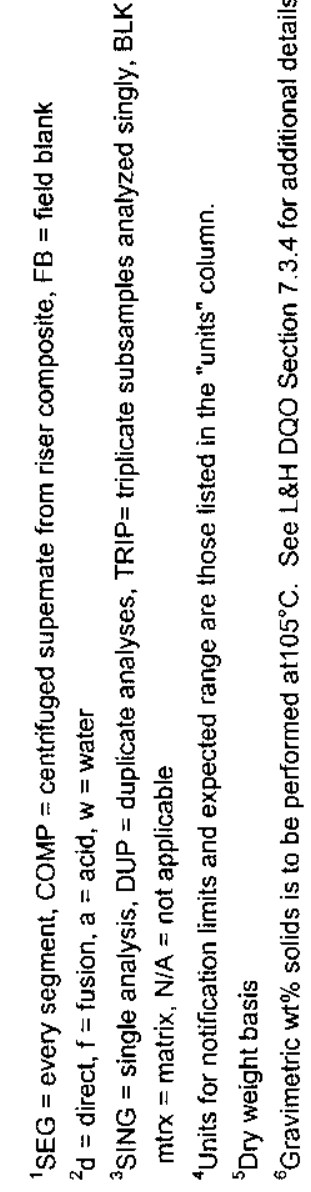




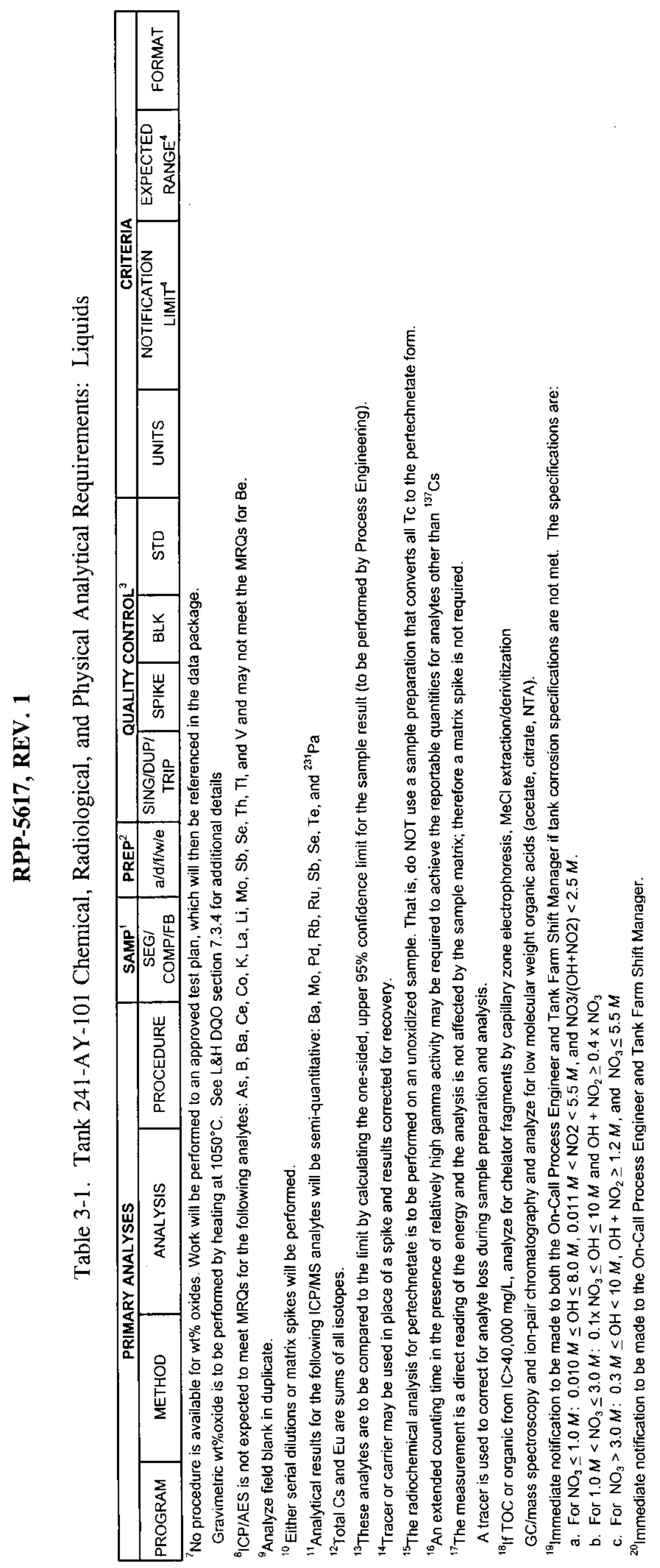




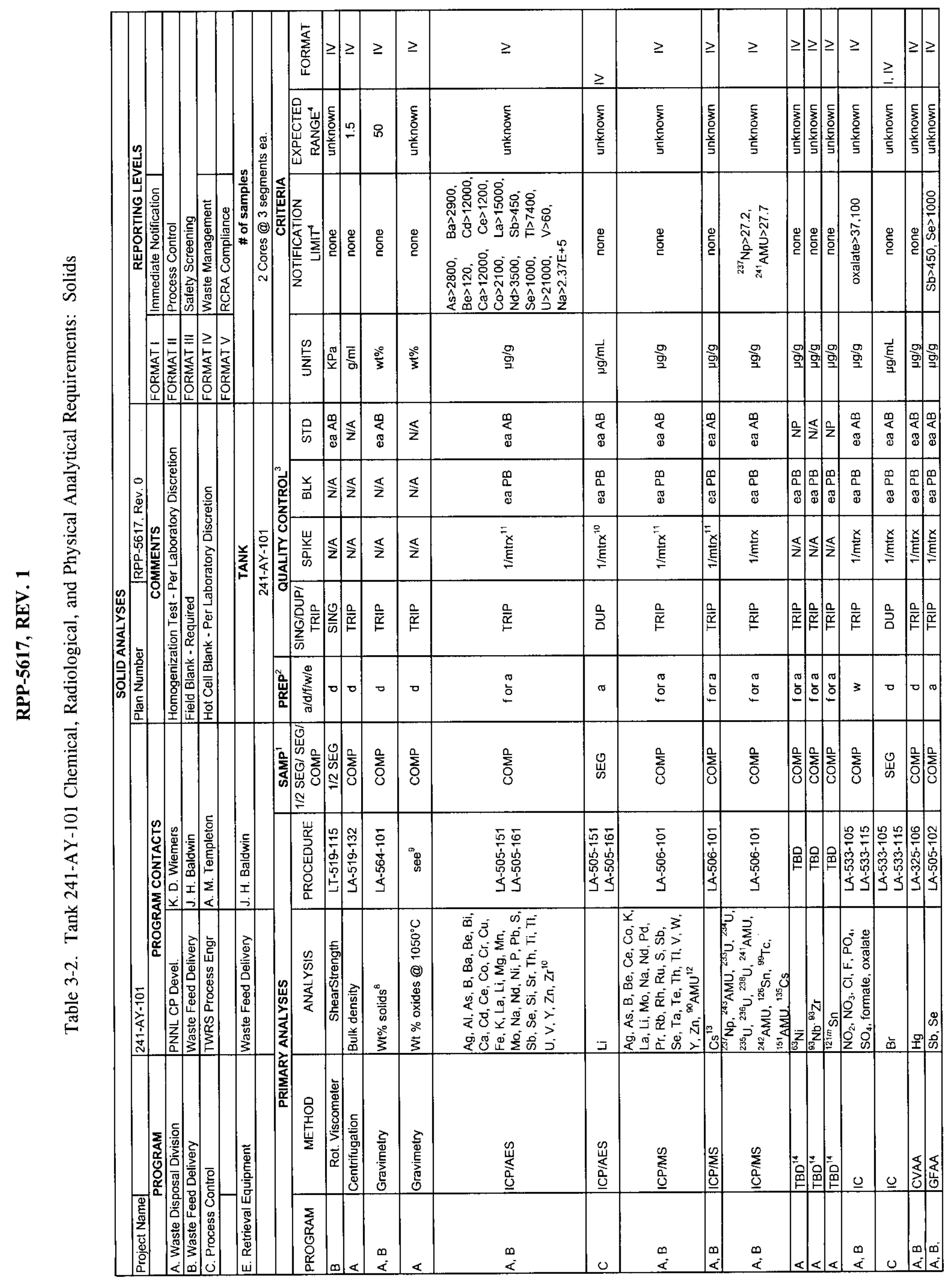




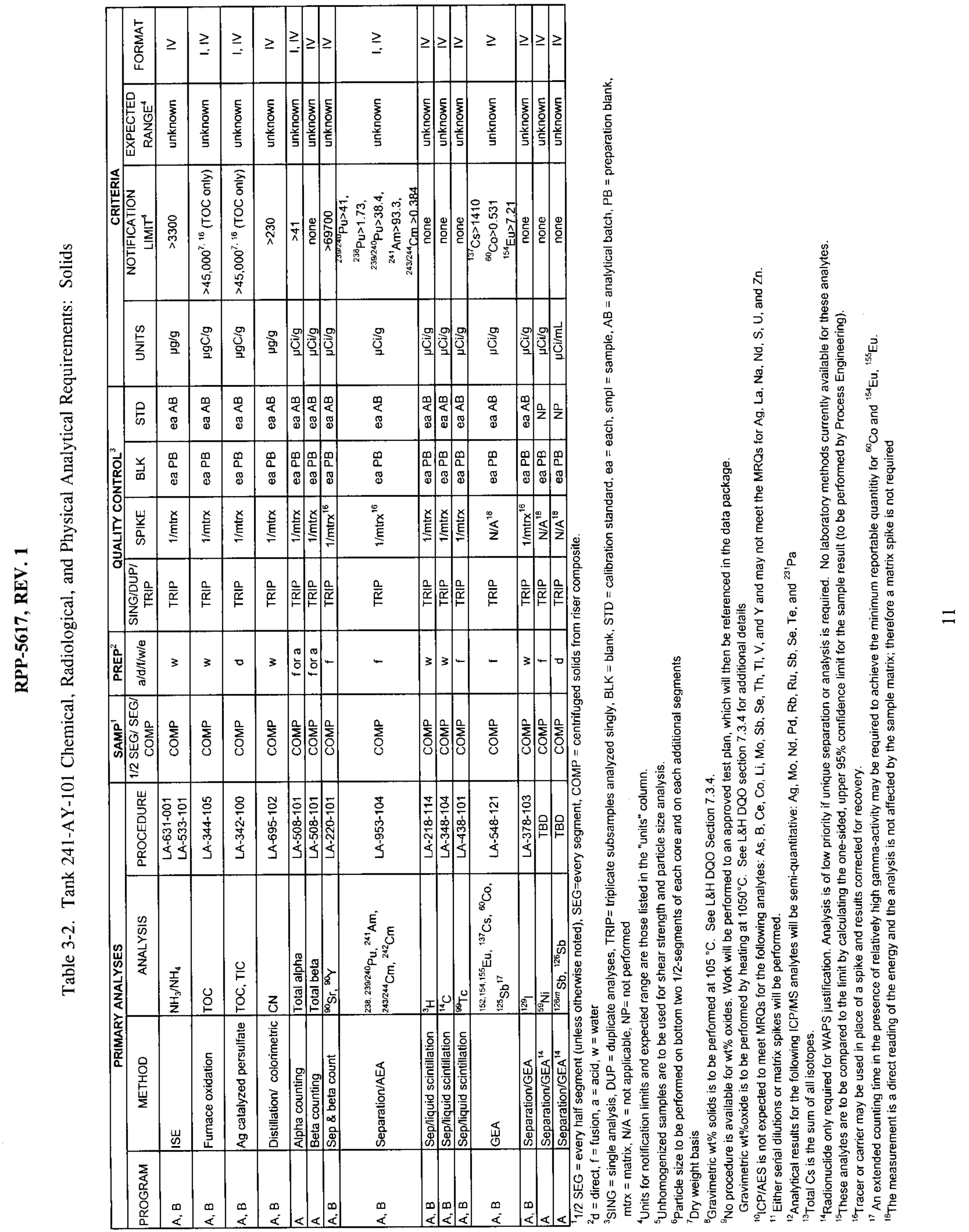


Table 3-3. Detection Limits and Minimum Reportable Quantities for Low-Activity Waste Liquids (4 sheets)

\begin{tabular}{|c|c|c|c|c|}
\hline Analyte & Method & $\begin{array}{c}\text { Estimated Quantitation } \\
\text { Limit/Minimum }^{(1)} \\
\text { Detectable Activity }^{(1)}\end{array}$ & $\begin{array}{c}\text { Minimum Reportable } \\
\text { Quantity (MRQ) }\end{array}$ & Units \\
\hline $\mathrm{Ag}$ & ICP/AES & $5.5 \mathrm{E}+00$ & $1.7 \mathrm{E}+01$ & $\mu \mathrm{g} / \mathrm{mL}$ \\
\hline $\mathrm{Al}$ & ICP/AES & $2.5 \mathrm{E}+01$ & $7.5 \mathrm{E}+01$ & $\mu \mathrm{g} / \mathrm{mL}$ \\
\hline As & ICP/MS & $7.5 \mathrm{E}-01(1.0 \mathrm{E}+00)$ & $2.3 \mathrm{E}+00(3.0 \mathrm{E}+00)$ & $\mu \mathrm{g} / \mathrm{mL}$ \\
\hline$B$ & ICP/MS & $7.5 \mathrm{E}-01$ & $2.3 \mathrm{E}+00$ & $\mu \mathrm{g} / \mathrm{mL}$ \\
\hline $\mathrm{Ba}$ & $\mathrm{ICP} / \mathrm{MS}$ & $7.5 \mathrm{E}-01(2.6 \mathrm{E}+01)$ & $2.3 \mathrm{E}+00(7.8 \mathrm{E}+01)$ & $\mu \mathrm{g} / \mathrm{mL}$ \\
\hline $\mathrm{Be}$ & $\mathrm{ICP} / \mathrm{MS}$ & $7.5 \mathrm{E}-01(3.0 \mathrm{E}+00)$ & $2.3 \mathrm{E}+00(9.9 \mathrm{E}+00)$ & $\mu \mathrm{g} / \mathrm{mL}$ \\
\hline $\mathrm{Bi}$ & ICP/AES & $5.5 \mathrm{E}+01$ & $1.7 \mathrm{E}+02$ & $\mu \mathrm{g} / \mathrm{mL}$ \\
\hline $\mathrm{Ca}$ & ICP/AES & $5.0 \mathrm{E}+01$ & $1.5 \mathrm{E}+02$ & $\mu \mathrm{g} / \mathrm{mL}$ \\
\hline $\mathrm{Cd}$ & $\mathrm{ICP} / \mathrm{AES}$ & $2.5 \mathrm{E}+00$ & $7.5 \mathrm{E}+00$ & $\mu \mathrm{g} / \mathrm{mL}$ \\
\hline $\mathrm{Ce}$ & ICP/MS & $7.5 \mathrm{E}-01$ & $2.3 \mathrm{E}+00$ & $\mu \mathrm{g} / \mathrm{mL}$ \\
\hline $\mathrm{Co}$ & $\mathrm{ICP} / \mathrm{MS}$ & $7.5 \mathrm{E}-01$ & $2.3 \mathrm{E}+00$ & $\mu \mathrm{g} / \mathrm{mL}$ \\
\hline $\mathrm{Cr}$ & $\mathrm{ICP} / \mathrm{AES}$ & $5.0 \mathrm{E}+00$ & $1.5 \mathrm{E}+01$ & $\mu \mathrm{g} / \mathrm{mL}$ \\
\hline $\mathrm{Cu}$ & ICP/AES & $5.5 \mathrm{E}+00$ & $1.7 \mathrm{E}+01$ & $\mu \mathrm{g} / \mathrm{mL}$ \\
\hline Cs, total & ICP/MS & $5.0 \mathrm{E}-01$ & $1.5 \mathrm{E} 00$ & $\mu \mathrm{g} / \mathrm{mL}$ \\
\hline Eu, total & ICP/MS & $2.0 \mathrm{E}+01$ & $6.0 \mathrm{E}+01$ & $\mu \mathrm{g} / \mathrm{mL}$ \\
\hline $\mathrm{Fe}$ & ICP/AES & $2.5 \mathrm{E}+01(3.0 \mathrm{E}+01)$ & $7.5 \mathrm{E}+01(9.9 \mathrm{E}+01)$ & $\mu \mathrm{g} / \mathrm{mL}$ \\
\hline $\mathrm{Hg}$ & CVAA & $5.0 \mathrm{E}-01$ & $1.5 \mathrm{E}+00(2.0 \mathrm{E}+00)$ & $\mu \mathrm{g} / \mathrm{mL}$ \\
\hline $\mathrm{K}$ & $\mathrm{ICP} / \mathrm{AES}$ & $2.5 \mathrm{E}+02$ & $7.5 \mathrm{E}+02(7.5 \mathrm{E}+01)$ & $\mu \mathrm{g} / \mathrm{mL}$ \\
\hline $\mathrm{La}$ & $\mathrm{ICP} / \mathrm{MS}$ & $7.5 \mathrm{E}-01(2.5 \mathrm{E}+01)$ & $2.3 \mathrm{E}+00(3.5 \mathrm{E}+01)$ & $\mu \mathrm{g} / \mathrm{mL}$ \\
\hline $\mathrm{Li}$ & ICP/MS & $7.5 \mathrm{E}-01$ & $2.3 \mathrm{E}+00$ & $\mu \mathrm{g} / \mathrm{mL}$ \\
\hline $\mathrm{Mg}$ & $\mathrm{ICP} / \mathrm{AES}$ & $5.5 \mathrm{E}+01 \quad(5.0 \mathrm{E}+01)$ & $1.7 \mathrm{E}+02(1.5 \mathrm{E}+02)$ & $\mu \mathrm{g} / \mathrm{mL}$ \\
\hline $\mathrm{Mn}$ & ICP/AES & $5.5 \mathrm{E}+00$ & $1.7 \mathrm{E}+01$ & $\mu \mathrm{g} / \mathrm{mL}$ \\
\hline Mo & ICP/MS & $7.5 \mathrm{E}-01(3.0 \mathrm{E}+01)$ & $2.3 \mathrm{E}+00(9.0 \mathrm{E}+01)$ & $\mu \mathrm{g} / \mathrm{mL}$ \\
\hline $\mathrm{Na}$ & $\mathrm{ICP} / \mathrm{AES}$ & $5.5 \mathrm{E}+01$ & $1.7 \mathrm{E}+02$ & $\mu \mathrm{g} / \mathrm{mL}$ \\
\hline $\mathrm{Nd}$ & ICP/AES & $5.5 \mathrm{E}+01$ & $1.7 \mathrm{E}+02$ & $\mu \mathrm{g} / \mathrm{mL}$ \\
\hline $\mathrm{Ni}$ & $\mathrm{ICP} / \mathrm{AES}$ & $1.0 \mathrm{E}+01$ & $3.0 \mathrm{E}+01$ & $\mu \mathrm{g} / \mathrm{mL}$ \\
\hline $\mathrm{P}$ & ICP/AES & $1.1 \mathrm{E}+02$ & $3.3 \mathrm{E}+02$ & $\mu \mathrm{g} / \mathrm{mL}$ \\
\hline $\mathrm{Pb}$ & ICP/AES & $9.9 \mathrm{E}+01$ & $3.0 \mathrm{E}+02$ & $\mu \mathrm{g} / \mathrm{mL}$ \\
\hline $\mathrm{Pd}$ & ICP/AES & $1.3 \mathrm{E}+02$ & $3.9 \mathrm{E}+02$ & $\mu \mathrm{g} / \mathrm{mL}$ \\
\hline $\operatorname{Pr}$ & $\mathrm{ICP} / \mathrm{MS}$ & $7.5 \mathrm{E}-01$ & $2.3 \mathrm{E}+00$ & $\mu \mathrm{g} / \mathrm{mL}$ \\
\hline
\end{tabular}


RPP-5617, Rev. 1

Table 3-3. Detection Limits and Minimum Reportable Quantities for Low-Activity Waste Liquids (4 sheets)

\begin{tabular}{|c|c|c|c|c|}
\hline Analyte & Method & $\begin{array}{c}\text { Estimated Quantitation } \\
\text { Limit/Minimum }^{(1)} \\
\text { Detectable Activity }^{(1)}\end{array}$ & $\begin{array}{c}\text { Minimum Reportable } \\
\text { Quantity (MRQ) }\end{array}$ & Units \\
\hline $\mathrm{Rb}$ & ICP/MS & $7.5 \mathrm{E}-01$ & $2.3 \mathrm{E}+00$ & $\mu \mathrm{g} / \mathrm{mL}$ \\
\hline $\mathrm{Rh}$ & ICP/AES & $6.0 \mathrm{E}+00$ & $1.8 \mathrm{E}+01$ & $\mu \mathrm{g} / \mathrm{mL}$ \\
\hline $\mathrm{Ru}$ & ICP/AES & $1.2 \mathrm{E}+01$ & $3.6 \mathrm{E}+01$ & $\mu \mathrm{g} / \mathrm{mL}$ \\
\hline$S$ & ICP/AES & $5.5 \mathrm{E}+01$ & $1.7 \mathrm{E}+02$ & $\mu \mathrm{g} / \mathrm{mL}$ \\
\hline $\mathrm{Sb}$ & ICP/MS & $7.5 \mathrm{E}-01(3.5 \mathrm{E}+01)$ & $2.3 \mathrm{E}+00(1.0 \mathrm{E}+02)$ & $\mu \mathrm{g} / \mathrm{mL}$ \\
\hline $\mathrm{Se}$ & $\mathrm{ICP} / \mathrm{MS}$ & $7.5 \mathrm{E}-01(5.5 \mathrm{E}+01)$ & $2.3 \mathrm{E}+00(1.7 \mathrm{E}+02)$ & $\mu \mathrm{g} / \mathrm{mL}$ \\
\hline $\mathrm{Si}$ & ICP/AES & $3.0 \mathrm{E}+01$ & $9.0 \mathrm{E}+01$ & $\mu \mathrm{g} / \mathrm{mL}$ \\
\hline $\mathrm{Sr}$ & ICP/AES & $5.5 \mathrm{E}+00$ & $1.7 \mathrm{E}+01$ & $\mu \mathrm{g} / \mathrm{mL}$ \\
\hline $\mathrm{Ta}$ & ICP/MS & $7.5 \mathrm{E}-01$ & $2.3 \mathrm{E}+00$ & $\mu \mathrm{g} / \mathrm{mL}$ \\
\hline $\mathrm{Te}$ & $\mathrm{ICP} / \mathrm{MS}$ & $7.5 \mathrm{E}-01$ & $2.3 \mathrm{E}+00$ & $\mu \mathrm{g} / \mathrm{mL}$ \\
\hline $\mathrm{Th}$ & $\mathrm{ICP} / \mathrm{MS}$ & $7.5 \mathrm{E}-01$ & $2.3 \mathrm{E}+00$ & $\mu \mathrm{g} / \mathrm{mL}$ \\
\hline $\mathrm{Ti}$ & ICP/AES & $5.5 \mathrm{E}+00$ & $1.7 \mathrm{E}+01$ & $\mu \mathrm{g} / \mathrm{mL}$ \\
\hline $\mathrm{Tl}$ & $\mathrm{ICP} / \mathrm{MS}$ & $7.5 \mathrm{E}-01$ & $2.3 \mathrm{E}+00$ & $\mu \mathrm{g} / \mathrm{mL}$ \\
\hline $\mathrm{U}$ & ICP/AES & $2.60 \mathrm{E}+02$ & $7.8 \mathrm{E}+02$ & $\mu \mathrm{g} / \mathrm{mL}$ \\
\hline $\mathrm{V}$ & $\mathrm{ICP} / \mathrm{MS}$ & $7.5 \mathrm{E}-01(3.0 \mathrm{E}+01)$ & $2.3 \mathrm{E}+00(9.0 \mathrm{E}+01)$ & $\mu \mathrm{g} / \mathrm{mL}$ \\
\hline W & $\mathrm{ICP} / \mathrm{MS}$ & $7.5 \mathrm{E}-01$ & $2.3 \mathrm{E}+00$ & $\mu \mathrm{g} / \mathrm{mL}$ \\
\hline $\mathrm{Y}\left({ }^{90} \mathrm{AMU}\right)$ & $\mathrm{ICP} / \mathrm{MS}$ & $7.5 \mathrm{E}-01(1.0 \mathrm{E}+02)$ & $2.3 \mathrm{E}+00(2.0 \mathrm{E}+02)$ & $\mu \mathrm{g} / \mathrm{mL}$ \\
\hline $\mathrm{Zn}$ & ICP/AES & $5.5 \mathrm{E}+00$ & $1.7 \mathrm{E}+01$ & $\mu \mathrm{g} / \mathrm{mL}$ \\
\hline $\mathrm{Zr}$ & ICP/AES & $5.5 \mathrm{E}+00$ & $1.7 \mathrm{E}+01$ & $\mu \mathrm{g} / \mathrm{mL}$ \\
\hline${ }^{3} \mathrm{H}$ & Liquid scint. & $7.0 \mathrm{E}-03$ & $2.1 \mathrm{E}-02$ & $\mu \mathrm{Ci} / \mathrm{mL}$ \\
\hline${ }^{14} \mathrm{C}$ & Liquid scint. & $2.4 \mathrm{E}-04$ & $7.2 \mathrm{E}-04$ & $\mu \mathrm{Ci} / \mathrm{mL}$ \\
\hline${ }^{60} \mathrm{Co}$ & GEA & $7.0 \mathrm{E}-04$ & $2.1 \mathrm{E}-03$ & $\mu \mathrm{Ci} / \mathrm{mL}$ \\
\hline${ }^{79} \mathrm{Se}$ & Liquid scint. & $3.0 \mathrm{E}-05$ & $9.0 \mathrm{E}-05$ & $\mu \mathrm{Ci} / \mathrm{mL}$ \\
\hline${ }^{89} \mathrm{Sr},{ }^{90} \mathrm{Sr}$ & Beta counting & $1.0 \mathrm{E}-02(5.0 \mathrm{E}-02)$ & $3.0 \mathrm{E}-02(1.5 \mathrm{E}-01)$ & $\mu \mathrm{Ci} / \mathrm{mL}$ \\
\hline${ }^{99} \mathrm{Tc}$ (total) & $\mathrm{ICP} / \mathrm{MS}$ & $5.0 \mathrm{E}-04$ & $1.5 \mathrm{E}-03$ & $\mu \mathrm{Ci} / \mathrm{mL}$ \\
\hline $\begin{array}{l}{ }^{99} \mathrm{Tc} \text { (pertech- } \\
\text { netate) }\end{array}$ & Liquid scint. & TBD & TBD & $\mu \mathrm{Ci} / \mathrm{mL}$ \\
\hline${ }^{125} \mathrm{Sb}$ & GEA & $5.6 \mathrm{E}-01$ & $1.7 \mathrm{E}+00$ & $\mu \mathrm{Ci} / \mathrm{mL}$ \\
\hline${ }^{126} \mathrm{Sn}$ & ICP/MS & $2.0 \mathrm{E}-03$ & $6.0 \mathrm{E}-03$ & $\mu \mathrm{Ci} / \mathrm{mL}$ \\
\hline${ }^{129} \mathrm{I}$ & GEA & $5.8 \mathrm{E}-06(3.5 \mathrm{E}-04)$ & $1.8 \mathrm{E}-05(1.1 \mathrm{E}-03)$ & $\mu \mathrm{Ci} / \mathrm{mL}$ \\
\hline${ }^{137} \mathrm{Cs}$ & GEA & $1.3 \mathrm{E}-01(3.0 \mathrm{E}+00)$ & $3.9 \mathrm{E}-01(9.0 \mathrm{E}+00)$ & $\mu \mathrm{Ci} / \mathrm{mL}$ \\
\hline${ }^{152} \mathrm{Eu}$ & GEA & TBD & TBD & $\mu \mathrm{Ci} / \mathrm{mL}$ \\
\hline
\end{tabular}


Table 3-3. Detection Limits and Minimum Reportable Quantities for Low-Activity Waste Liquids (4 sheets)

\begin{tabular}{|c|c|c|c|c|}
\hline Analyte & Method & $\begin{array}{c}\text { Estimated Quantitation } \\
\text { Limit/Minimum } \\
\text { Detectable Activity }^{(1)} \\
\end{array}$ & $\begin{array}{l}\text { Minimum Reportable } \\
\text { Quantity (MRQ) }^{(1)}\end{array}$ & Units \\
\hline${ }^{154} \mathrm{Eu}$ & GEA & $6.5 \mathrm{E}-03$ & $2.0 \mathrm{E}-02(2.0 \mathrm{E}-03)$ & $\mu \mathrm{Ci} / \mathrm{mL}$ \\
\hline${ }^{155} \mathrm{Eu}$ & GEA & $3.0 \mathrm{E}-02$ & $9.0 \mathrm{E}-02$ & $\mu \mathrm{Ci} / \mathrm{mL}$ \\
\hline${ }^{231} \mathrm{~Pa}$ & $\mathrm{ICP} / \mathrm{MS}$ & TBD & TBD & $\mu \mathrm{Ci} / \mathrm{mL}$ \\
\hline${ }^{233} \mathrm{U}$ & ICP/MS & $1.4 \mathrm{E}-04(6.0 \mathrm{E}-04)$ & $4.2 \mathrm{E}-04(1.8 \mathrm{E}-03)$ & $\mu \mathrm{Ci} / \mathrm{mL}$ \\
\hline${ }^{234} \mathrm{U}$ & $\mathrm{ICP} / \mathrm{MS}$ & $4.4 \mathrm{E}-05(4.0 \mathrm{E}-08)$ & 1.2E-04 (1.2E-07) & $\mu \mathrm{Ci} / \mathrm{mL}$ \\
\hline${ }^{235} \mathrm{U}$ & ICP/MS & $1.5 \mathrm{E}-08(1.1 \mathrm{E}-06)$ & $4.5 \mathrm{E}-08(4.5 \mathrm{E}-06)$ & $\mu \mathrm{Ci} / \mathrm{mL}$ \\
\hline${ }^{236} \mathrm{U}$ & ICP/MS & $4.5 \mathrm{E}-07$ & $1.4 \mathrm{E}-06$ & $\mu \mathrm{Ci} / \mathrm{mL}$ \\
\hline${ }^{237} \mathrm{~Np}$ & ICP/MS & $1.3 \mathrm{E}-05(9.1 \mathrm{E}-03)$ & $3.9 \mathrm{E}-05(2.7 \mathrm{E}-02)$ & $\mu \mathrm{Ci} / \mathrm{mL}$ \\
\hline${ }^{238} \mathrm{Pu}$ & AEA & $3.4 \mathrm{E}-03(3.2 \mathrm{E}-03)$ & $1.0 \mathrm{E}-02(9.6 \mathrm{E}-03)$ & $\mu \mathrm{Ci} / \mathrm{mL}$ \\
\hline${ }^{238} \mathrm{U}$ & ICP/MS & $2.4 \mathrm{E}-09(1.7 \mathrm{E}-07)$ & $7.2 \mathrm{E}-09(5.0 \mathrm{E}-07)$ & $\mu \mathrm{Ci} / \mathrm{mL}$ \\
\hline${ }^{239} \mathrm{Pu}$ & $\mathrm{AEA}$ & $3.4 \mathrm{E}-03(3.20 \mathrm{E}-03)$ & $1.0 \mathrm{E}-02(9.6 \mathrm{E}-03)$ & $\mu \mathrm{Ci} / \mathrm{mL}$ \\
\hline${ }^{240} \mathrm{Pu}$ & AEA & $1.7 \mathrm{E}-02(3.2 \mathrm{E}-03)$ & $5.1 \mathrm{E}-02(9.6 \mathrm{E}-03)$ & $\mu \mathrm{Ci} / \mathrm{mL}$ \\
\hline${ }^{241} \mathrm{Pu}$ & NS & NS (3.2E-03) & NS (9.6E-03) & $\mu \mathrm{Ci} / \mathrm{mL}$ \\
\hline $\begin{array}{l}{ }^{241} \mathrm{Pu} / \mathrm{Am} \\
\left({ }^{241} \mathrm{AMU}\right)\end{array}$ & ICP/MS & $1.6 \mathrm{E}+00$ & $4.8 \mathrm{E}+01$ & $\mu \mathrm{Ci} / \mathrm{mL}$ \\
\hline${ }^{242} \mathrm{Pu}\left({ }^{242} \mathrm{AMU}\right)$ & ICP/MS & $1.0 \mathrm{E}-02(3.2 \mathrm{E}-03)$ & $3.0 \mathrm{E}-02(9.6 \mathrm{E}-03)$ & $\mu \mathrm{Ci} / \mathrm{mL}$ \\
\hline${ }^{241} \mathrm{Am}$ & AEA & 1.0E-02(2.4E-04) & $3.0 \mathrm{E}-02(7.2 \mathrm{E}-04)$ & $\mu \mathrm{Ci} / \mathrm{mL}$ \\
\hline${ }^{242} \mathrm{Cm}$ & AEA & NS & NS & $\mu \mathrm{Ci} / \mathrm{mL}$ \\
\hline${ }^{243} \mathrm{Am}\left({ }^{243} \mathrm{AMU}\right)$ & ICP/MS & $3.2 \mathrm{E}-03$ & $9.6 \mathrm{E}-03$ & $\mu \mathrm{Ci} / \mathrm{mL}$ \\
\hline${ }^{243+244} \mathrm{Cm}$ & AEA & $5.0 \mathrm{E}-02$ & $1.5 \mathrm{E}-01$ & $\mu \mathrm{Ci} / \mathrm{mL}$ \\
\hline $\mathrm{NH}_{4} / \mathrm{NH}_{3}$ & ISE & $4.5 \mathrm{E}+01$ & $1.4 \mathrm{E}+02$ & $\mu \mathrm{g} / \mathrm{mL}$ \\
\hline $\mathrm{Cl}$ & IC & $1.0 \mathrm{E}+02$ & $3.0 \mathrm{E}+02$ & $\mu \mathrm{g} / \mathrm{mL}$ \\
\hline $\mathrm{CN}$ & $\begin{array}{l}\text { Distillation/ } \\
\text { colorimetric }\end{array}$ & $1.5 \mathrm{E}+00$ & $4.5 \mathrm{E}+00$ & $\mu \mathrm{g} / \mathrm{mL}$ \\
\hline $\mathrm{F}$ & IC & $5.0 \mathrm{E}+01$ & $1.5 \mathrm{E}+02$ & $\mu \mathrm{g} / \mathrm{mL}$ \\
\hline $\mathrm{NO}_{2}$ & IC & $7.5 \mathrm{E}+02$ & $2.3 \mathrm{E}+03$ & $\mu \mathrm{g} / \mathrm{mL}$ \\
\hline $\mathrm{NO}_{3}$ & IC & $1.0 \mathrm{E}+03$ & $3.0 \mathrm{E}+03$ & $\mu \mathrm{g} / \mathrm{mL}$ \\
\hline $\mathrm{OH}$ & Titration & $2.5 \mathrm{E}+04$ & $7.5 \mathrm{E}+04$ & $\mu \mathrm{g} / \mathrm{mL}$ \\
\hline oxalate & IC & $6.0 \mathrm{E}+02$ & $1.8 \mathrm{E}+03$ & $\mu \mathrm{g} / \mathrm{mL}$ \\
\hline $\mathrm{PO}_{4}$ & $\mathrm{IC}$ & $7.5 \mathrm{E}+02$ & $2.3 \mathrm{E}+03(2.5 \mathrm{E}+03)$ & $\mu \mathrm{g} / \mathrm{mL}$ \\
\hline $\mathrm{SO}_{4}$ & IC & $7.7 \mathrm{E}+02$ & $2.3 \mathrm{E}+03$ & $\mu \mathrm{g} / \mathrm{mL}$ \\
\hline total alpha & Prop. counting & $7.5 \mathrm{E}-02$ & $2.3 \mathrm{E}-01$ & $\mu \mathrm{Ci} / \mathrm{mL}$ \\
\hline total beta & Beta counting & TBD & TBD & $\mu \mathrm{Ci} / \mathrm{mL}$ \\
\hline
\end{tabular}


RPP-5617, Rev. 1

Table 3-3. Detection Limits and Minimum Reportable Quantities for Low-Activity Waste Liquids (4 sheets)

\begin{tabular}{|l|l|c|c|c|}
\hline \multicolumn{1}{|c|}{ Analyte } & \multicolumn{1}{|c|}{ Method } & $\begin{array}{c}\text { Estimated Quantitation } \\
\text { Limit/Minimum } \\
\text { Detectable Activity }\end{array}$ & $\begin{array}{c}\text { Minimum Reportable } \\
\text { Quantity (MRQ) }\end{array}$ & Units \\
\hline $\begin{array}{l}\text { total inorganic } \\
\text { carbon }\end{array}$ & $\begin{array}{l}\text { Persulfate/ } \\
\text { furnace oxidation }\end{array}$ & $5.0 \mathrm{E}+01$ & $1.5 \mathrm{E}+02$ & $\mu \mathrm{g} / \mathrm{mL}$ \\
\hline $\begin{array}{l}\text { total organic } \\
\text { carbon }\end{array}$ & $\begin{array}{l}\text { Persulfate/ } \\
\text { furnace oxidation }\end{array}$ & $5.0 \mathrm{E}+02$ & $1.5 \mathrm{E}+03$ & $\mu \mathrm{g} / \mathrm{mL}$ \\
\hline
\end{tabular}

Notes:

$\mathrm{AEA}=$ alpha energy analysis

CVAA $=$ cold vapor atomic absorption

$\mathrm{GEA}=$ gamma energy analysis

IC $=$ ion chromatography

ICP/AES = inductively coupled plasma/atomic emission spectroscopy

$\mathrm{ICP} / \mathrm{MS}=$ inductively coupled plasma /mass spectrometry

ISE $=$ ion-specific electrode

liquid scint. = liquid scintillation

NS = not specified

prop. counting $=$ proportional counting

$\mathrm{TBD}=$ to be determined

' Detection limits and MRQ's for the L\&H DQO. Where LAW DQO requirements differ from those listed, the corresponding HLW or LAW requirement is shown in parentheses. The LAW requirements apply only if testing shows that dilution is not needed to meet waste transfer requirements. If process testing determines that dilution is required for transfer, requirements for analysis of the diluted waste will be as per the test plan.

${ }^{2}$ NS not stated for the L\&H DQO. In the event that testing shows that dilution is not required to meet waste transfer requirements, then the LAW MRQ's apply. If process testing determines that dilution is required for transfer, requirements for analysis of the diluted waste will be as per the test plan. 
Table 3-4. Detection Limits and Minimum Reportable Quantities for High Level Waste Solids (4sheets)

\begin{tabular}{|c|c|c|c|c|}
\hline Analyte & Method & $\begin{array}{c}\text { Estimated } \\
\text { Quantitation } \\
\text { Limit/Minimum } \\
\text { Detectable Activity } \\
(1)\end{array}$ & $\begin{array}{c}\text { Minimum Reportable } \\
\text { Quantity (MRQ) }^{(1)}\end{array}$ & $\begin{array}{c}\text { Units } \\
\text { (per gram } \\
\text { dried solids) }\end{array}$ \\
\hline $\mathrm{Ag}$ & ICP/AES & $300(50)$ & $900(900140)$ & $\mu \mathrm{g} / \mathrm{g}$ \\
\hline $\mathrm{Al}$ & $\mathrm{ICP} / \mathrm{AES}$ & $1200(110)$ & $3600(330)$ & $\mu \mathrm{g} / \mathrm{g}$ \\
\hline As & ICP/MS & $20(10)$ & $60(30)$ & $\mu \mathrm{g} / \mathrm{g}$ \\
\hline B & ICP/MS & $10(1)$ & $30(3)$ & $\mu \mathrm{g} / \mathrm{g}$ \\
\hline $\mathrm{Ba}$ & ICP/AES & 200 & 600 & $\mu \mathrm{g} / \mathrm{g}$ \\
\hline $\mathrm{Be}$ & ICP/MS & 10 & 30 & $\mu \mathrm{g} / \mathrm{g}$ \\
\hline$\overline{B i}$ & ICP/AES & 2000 & 6000 & $\mu \mathrm{g} / \mathrm{g}$ \\
\hline $\mathrm{Ca}$ & ICP/AES & $2000(62)$ & $6000(180)$ & $\mu \mathrm{g} / \mathrm{g}$ \\
\hline $\mathrm{Cd}$ & ICP/AES & $300(4)$ & $900(11)$ & $\mu \mathrm{g} / \mathrm{g}$ \\
\hline $\mathrm{Ce}$ & ICP/MS & 2 & 6 & $\mu \mathrm{g} / \mathrm{g}$ \\
\hline Co & ICP/MS & $2(1)$ & $6(3)$ & $\mu \mathrm{g} / \mathrm{g}$ \\
\hline $\mathrm{Cr}$ & ICP/AES & $400(40)$ & $1200(120)$ & $\mu \mathrm{g} / \mathrm{g}$ \\
\hline Cs & $\mathrm{NS}^{2}$ & $\mathrm{NS}^{2}(2.0 \mathrm{E}+00)$ & $\mathrm{NS}^{2}(6.0 \mathrm{E}+00)$ & $\mu \mathrm{g} / \mathrm{g}$ \\
\hline $\mathrm{Cu}$ & ICP/AES & $200(6.5)$ & $600(18)$ & $\mu \mathrm{g} / \mathrm{g}$ \\
\hline $\mathrm{F}$ & IC & 2500 & 7500 & $\mu \mathrm{g} / \mathrm{g}$ \\
\hline $\mathrm{Fe}$ & ICP/AES & $400(50)$ & $1200(140)$ & $\mu \mathrm{g} / \mathrm{g}$ \\
\hline $\mathrm{Hg}$ & CVAA & 0.5 & 1.5 & $\mu \mathrm{g} / \mathrm{g}$ \\
\hline $\mathrm{K}$ & ICP/MS & $2000(500)$ & $6000(1500)$ & $\mu \mathrm{g} / \mathrm{g}$ \\
\hline $\mathrm{La}$ & ICP/AES & $1000(20)$ & $3000(60)$ & $\mu \mathrm{g} / \mathrm{g}$ \\
\hline $\mathrm{Li}$ & ICP/MS & 10 & 30 & $\mu \mathrm{g} / \mathrm{g}$ \\
\hline $\mathrm{Mg}$ & ICP/AES & $1800(180)$ & $5400(540)$ & $\mu \mathrm{g} / \mathrm{g}$ \\
\hline $\mathrm{Mn}$ & ICP/AES & 100 & 300 & $\mu \mathrm{g} / \mathrm{g}$ \\
\hline Mo & ICP/MS & 2 & 6 & $\mu \mathrm{g} / \mathrm{g}$ \\
\hline $\mathrm{Na}$ & ICP/AES & $1800(50)$ & $5400(150)$ & $\mu \mathrm{g} / \mathrm{g}$ \\
\hline $\mathrm{Nd}$ & ICP/AES & $1000(26)$ & $3000(77)$ & $\mu \mathrm{g} / \mathrm{g}$ \\
\hline $\mathrm{Ni}$ & ICP/AES & $600(55)$ & $1800(160)$ & $\mu \mathrm{g} / \mathrm{g}$ \\
\hline $\mathrm{P}$ & ICP/AES & $2000(200)$ & $6000(600)$ & $\mu \mathrm{g} / \mathrm{g}$ \\
\hline $\mathrm{Pb}$ & ICP/AES & $1200(200)$ & $3600(600)$ & $\mu \mathrm{g} / \mathrm{g}$ \\
\hline$\overline{\mathrm{Pd}}$ & $\mathrm{ICP} / \mathrm{MS}$ & $10(1)$ & $30(3)$ & $\mu \mathrm{g} / \mathrm{g}$ \\
\hline $\mathrm{Pr}$ & ICP/MS & 2 & 6 & $\mu \mathrm{g} / \mathrm{g}$ \\
\hline $\mathrm{Pu}$ & $\mathrm{ICP} / \mathrm{MS}$ & $2(8)$ & $6(24)$ & $\mu \mathrm{g} / \mathrm{g}$ \\
\hline $\mathrm{Rb}$ & ICP/MS & 2 & 6 & $\mu \mathrm{g} / \mathrm{g}$ \\
\hline $\mathrm{Rh}$ & ICP/MS & 2 & 6 & $\mu \mathrm{g} / \mathrm{g}$ \\
\hline $\mathrm{Ru}$ & ICP/MS & $4(1)$ & $12(3)$ & $\mu \mathrm{g} / \mathrm{g}$ \\
\hline$S$ & ICP/MS & $\mathrm{NS}^{2}(40)$ & $\mathrm{NS}^{2}(120)$ & $\mu \mathrm{g} / \mathrm{g}$ \\
\hline $\mathrm{Sb}$ & ICP/MS & 4 & 12 & $\mu \mathrm{g} / \mathrm{g}$ \\
\hline $\mathrm{Se}$ & ICP/MS & 100 & 300 & $\mu \mathrm{g} / \mathrm{g}$ \\
\hline
\end{tabular}


Table 3-4. Detection Limits and Minimum Reportable Quantities for High Level Waste Solids (4sheets)

\begin{tabular}{|c|c|c|c|c|}
\hline Analyte & Method & $\begin{array}{c}\text { Estimated } \\
\text { Quantitation } \\
\text { Limit/Minimum } \\
\text { Detectable Activity }{ }^{(1)}\end{array}$ & $\begin{array}{l}\text { Minimum Reportable } \\
\text { Quantity (MRQ) }^{(1)}\end{array}$ & $\begin{array}{c}\text { Units } \\
\text { (per gram } \\
\text { dried solids) }\end{array}$ \\
\hline $\mathrm{Si}$ & ICP/AES & $10000(1000)$ & $30000(3000)$ & $\mu \mathrm{g} / \mathrm{g}$ \\
\hline $\mathrm{Sr}$ & $\mathrm{ICP} / \mathrm{AES}$ & 100 & 300 & $\mu \mathrm{g} / \mathrm{g}$ \\
\hline $\mathrm{Ta}$ & $\mathrm{ICP} / \mathrm{MS}$ & 2 & 6 & $\mu \mathrm{g} / \mathrm{g}$ \\
\hline Tc & $\mathrm{ICP} / \mathrm{MS}$ & (2) & (6) & $\mu \mathrm{g} / \mathrm{g}$ \\
\hline $\mathrm{Te}$ & ICP/MS & $6(2)$ & $18(6)$ & $\mu \mathrm{g} / \mathrm{g}$ \\
\hline Th & $\mathrm{ICP} / \mathrm{MS}$ & $2(200)$ & $6(600)$ & $\mu \mathrm{g} / \mathrm{g}$ \\
\hline $\mathrm{Ti}$ & ICP/AES & $200(50)$ & $600(150)$ & $\mu \mathrm{g} / \mathrm{g}$ \\
\hline $\mathrm{Tl}$ & $\mathrm{ICP} / \mathrm{MS}$ & $2(200)$ & $6(600)$ & $\mu \mathrm{g} / \mathrm{g}$ \\
\hline $\mathrm{U}$ & $\mathrm{NS}$ & $\mathrm{NS}(2.0 \mathrm{E}+02)$ & $\mathrm{NS}(6.0 \mathrm{E}+02)$ & $\mu \mathrm{g} / \mathrm{g}$ \\
\hline $\mathrm{V}$ & ICP/MS & $2(0.02)$ & $6(0.06)$ & $\mu \mathrm{g} / \mathrm{g}$ \\
\hline $\mathrm{W}$ & ICP/MS & 2 & 6 & $\mu \mathrm{g} / \mathrm{g}$ \\
\hline $\mathrm{Y}$ & ICP/MS & $2(90)$ & $6(270)$ & $\mu \mathrm{g} / \mathrm{g}$ \\
\hline $\mathrm{Zn}$ & ICP/AES & $400(2)$ & $1200(6)$ & $\mu \mathrm{g} / \mathrm{g}$ \\
\hline $\mathrm{Zr}$ & ICP/AES & 200 & 600 & $\mu \mathrm{g} / \mathrm{g}$ \\
\hline $\mathrm{Cl}$ & IC & 75 & 225 & $\mu \mathrm{g} / \mathrm{g}$ \\
\hline $\mathrm{CN}^{-}$ & $\mathrm{CN}^{-}$analysis & 1.0 & 3 & $\mu \mathrm{g} / \mathrm{g}$ \\
\hline $\mathrm{CO}_{3}^{-2}$ & $\begin{array}{l}\text { Persulfate/furnace } \\
\text { oxidation }\end{array}$ & NS (10) & NS (30) & $\mu \mathrm{g} / \mathrm{g}$ \\
\hline $\mathrm{NH}_{3}$ & ISE & 20 & 60 & $\mu \mathrm{g} / \mathrm{g}$ \\
\hline $\mathrm{NO}_{2}^{-}$ & IC & 150 & 450 & $\mu \mathrm{g} / \mathrm{g}$ \\
\hline $\mathrm{NO}_{3}{ }^{-}$ & IC & 150 & 450 & $\mu \mathrm{g} / \mathrm{g}$ \\
\hline TOC & $\begin{array}{l}\text { Persulfate/furnace } \\
\text { oxidation }\end{array}$ & 20 & 60 & $\mu \mathrm{g} \mathrm{C} / \mathrm{g}$ \\
\hline${ }^{3} \mathrm{H}$ & $\mathrm{NS}$ & NS (5.0E-03) & NS (1.5E-02) & $\mu \mathrm{Ci} / \mathrm{g}$ \\
\hline${ }^{14} \mathrm{C}$ & Liquid scint. & $2.0 \mathrm{E}-04(6.0 \mathrm{E}-04)$ & $6.0 \mathrm{E}-04(1.8 \mathrm{E}-03)$ & $\mu \mathrm{Ci} / \mathrm{g}$ \\
\hline${ }^{59} \mathrm{Ni}^{3}$ & Sep./GEA & $1.0 \mathrm{E}-02$ & $3.0 \mathrm{E}-02$ & $\mu \mathrm{Ci} / \mathrm{g}$ \\
\hline${ }^{60} \mathrm{Co}$ & GEA & $4.0 \mathrm{E}-02(4.0 \mathrm{E}-03)$ & $1.2 \mathrm{E}-01(1.2 \mathrm{E}-02)$ & $\mu \mathrm{Ci} / \mathrm{g}$ \\
\hline${ }^{63} \mathrm{Ni}$ & Liquid scint. & $2.0 \mathrm{E}-03$ & $6.0 \mathrm{E}-03$ & $\mu \mathrm{Ci} / \mathrm{g}$ \\
\hline${ }^{90} \mathrm{Sr}^{4}$ & Beta counting & $7.0 \mathrm{E}+00$ & $2.1 \mathrm{E}+01$ & $\mu \mathrm{Ci} / \mathrm{g}$ \\
\hline${ }^{90} \mathrm{Y}^{4}$ & Beta counting & $7.0 \mathrm{E}+00$ & $2.1 \mathrm{E}+01$ & $\mu \mathrm{Ci} / \mathrm{g}$ \\
\hline${ }^{93} \mathrm{Zr}^{5}$ & Liquid scint. & $2.0 \mathrm{E}-03$ & $6.0 \mathrm{E}-03$ & $\mu \mathrm{Ci} / \mathrm{g}$ \\
\hline $\begin{array}{l}{ }^{93 \mathrm{~m}} \mathrm{Nb} \\
\left({ }^{93} \mathrm{AMU}\right)^{5}\end{array}$ & ICP/MS & 4 & 12 & $\mu \mathrm{Ci} / \mathrm{g}$ \\
\hline${ }^{99} \mathrm{Tc}$ & ICP/MS & $2.0 \mathrm{E}+00$ & $6.0 \mathrm{E}+00$ & $\mu \mathrm{Ci} / \mathrm{g}$ \\
\hline${ }^{121 \mathrm{~m}} \mathrm{Sn}$ & Sep./GEA & $9.0 \mathrm{E}-02$ & $2.7 \mathrm{E}-01$ & $\mu \mathrm{Ci} / \mathrm{g}$ \\
\hline${ }^{125} \mathrm{Sb}^{6}$ & GEA & $2.0 \mathrm{E}+00$ & $6.0 \mathrm{E}+00$ & $\mu \mathrm{Ci} / \mathrm{g}$ \\
\hline${ }^{125 \mathrm{~m}} \mathrm{Te}^{6}$ & GEA & $2.0 \mathrm{E}+00$ & $6.0 \mathrm{E}+00$ & $\mu \mathrm{Ci} / \mathrm{g}$ \\
\hline
\end{tabular}


RPP-5617, Rev. 1

Table 3-4. Detection Limits and Minimum Reportable Quantities for High Level Waste Solids (4sheets)

\begin{tabular}{|c|c|c|c|c|}
\hline Analyte & Method & $\begin{array}{c}\text { Estimated } \\
\text { Quantitation } \\
\text { Limit/Minimum } \\
\text { Detectable Activity }^{(1)}\end{array}$ & $\begin{array}{c}\text { Minimum Reportable } \\
\text { Quantity (MRQ) }^{(1)}\end{array}$ & $\begin{array}{c}\text { Units } \\
\text { (per gram } \\
\text { dried solids) }\end{array}$ \\
\hline${ }^{126 m} \mathrm{Sb}^{3,7}$ & Sep./GEA & $6.0 \mathrm{E}-03$ & $1.8 \mathrm{E}-02$ & $\mu \mathrm{Ci} / \mathrm{g}$ \\
\hline${ }^{126} \mathrm{Sb}^{3,7}$ & Sep./GEA & $6.0 \mathrm{E}-03$ & $1.8 \mathrm{E}-02$ & $\mu \mathrm{Ci} / \mathrm{g}$ \\
\hline${ }^{126} \mathrm{Sn}^{7}$ & ICP/MS & $6.0 \mathrm{E}-03(2.0 \mathrm{E}-02)$ & $1.8 \mathrm{E}-02(6.0 \mathrm{E}-02)$ & $\mu \mathrm{Ci} / \mathrm{g}$ \\
\hline${ }^{129} \mathrm{I}$ & ICP/MS & 10 & 30 & $\mu \mathrm{Ci} / \mathrm{g}$ \\
\hline${ }^{135} \mathrm{Cs}$ & ICP/MS & 2 & 6 & $\mu \mathrm{Ci} / \mathrm{g}$ \\
\hline${ }^{137 m} \mathrm{Ba}^{8}$ & GEA & $3.0 \mathrm{E}-02$ & $9.0 \mathrm{E}-02$ & $\mu \mathrm{Ci} / \mathrm{g}$ \\
\hline${ }^{137} \mathrm{Cs}^{8}$ & GEA & $3.0 \mathrm{E}-02(2.0 \mathrm{E}-02)$ & $9.0 \mathrm{E}-02(6.0 \mathrm{E}-02)$ & $\mu \mathrm{Ci} / \mathrm{g}$ \\
\hline${ }^{151} \mathrm{AMU}$ & ICP/MS & 2 & 6 & $\mu \mathrm{Ci} / \mathrm{g}$ \\
\hline${ }^{152} \mathrm{Eu}$ & GEA & $2.0 \mathrm{E}+00$ & $6.0 \mathrm{E}+00(2 .-\mathrm{E}+00)$ & $\mu \mathrm{Ci} / \mathrm{g}$ \\
\hline${ }^{154} \mathrm{Eu}$ & GEA & $1.0 \mathrm{E}-01(2.0 \mathrm{E}-02)$ & $3.0 \mathrm{E}-01(6.0 \mathrm{E}-02)$ & $\mu \mathrm{Ci} / \mathrm{g}$ \\
\hline${ }^{155} \mathrm{Eu}$ & GEA & $2.0 \mathrm{E}+00(2.0 \mathrm{E}-02)$ & $6.0 \mathrm{E}+00(6.0 \mathrm{E}-02)$ & $\mu \mathrm{Ci} / \mathrm{g}$ \\
\hline${ }^{233} \mathrm{U}$ & ICP/MS & $0.2(2.0 \mathrm{E}+00)$ & $0.6(6.0 \mathrm{E}+00)$ & $\mu \mathrm{Ci} / \mathrm{g}$ \\
\hline${ }^{234} \mathrm{U}$ & ICP/MS & 2 & 6 & $\mu \mathrm{Ci} / \mathrm{g}$ \\
\hline${ }^{235} \mathrm{U}$ & ICP/MS & 2 & 6 & $\mu \mathrm{Ci} / \mathrm{g}$ \\
\hline${ }^{236} \mathrm{U}$ & ICP/MS & 2 & 6 & $\mu \mathrm{Ci} / \mathrm{g}$ \\
\hline${ }^{237} \mathrm{~Np}$ & $\mathrm{ICP} / \mathrm{MS}$ & $2(6.0 \mathrm{E}-01)$ & $6(1.8 \mathrm{E}+00)$ & $\mu \mathrm{Ci} / \mathrm{g}$ \\
\hline${ }^{238} \mathrm{Pu}$ & Sep./AEA & $2.0 \mathrm{E}-02(2.0 \mathrm{E}-05)$ & $6.0 \mathrm{E}-02(6.0 \mathrm{E}-05)$ & $\mu \mathrm{Ci} / \mathrm{g}$ \\
\hline $238 \mathrm{U}$ & ICP/MS & 2 & 6 & $\mu \mathrm{Ci} / \mathrm{g}$ \\
\hline${ }^{239} \mathrm{Pu}^{9}$ & Sep./AEA & $2.0 \mathrm{E}-02(2.0 \mathrm{E}+00)$ & $6.0 \mathrm{E}-02(6.0 \mathrm{E}+00)$ & $\mu \mathrm{Ci} / \mathrm{g}$ \\
\hline${ }^{240} \mathrm{Pu}^{9}$ & Sep./AEA & $2.0 \mathrm{E}-02$ & $6.0 \mathrm{E}-02$ & $\mu \mathrm{Ci} / \mathrm{g}$ \\
\hline${ }^{241} \mathrm{Am}$ & Sep./AEA & $6.0 \mathrm{E}-03(4.0 \mathrm{E}-04)$ & $1.8 \mathrm{E}-02(1.2 \mathrm{E}-03)$ & $\mu \mathrm{Ci} / \mathrm{g}$ \\
\hline${ }^{24 !} \mathrm{AMU}$ & $\mathrm{ICP} / \mathrm{MS}$ & 2 & 6 & $\mu \mathrm{Ci} / \mathrm{g}$ \\
\hline${ }^{242} \mathrm{Cm}^{10}$ & Sep./AEA & $4.0 \mathrm{E}-03$ & $1.2 \mathrm{E}-02$ & $\mu \mathrm{Ci} / \mathrm{g}$ \\
\hline${ }^{242 \mathrm{~m}} \mathrm{Am}^{10}$ & Sep./AEA & $4.0 \mathrm{E}-03$ & $1.2 \mathrm{E}-02$ & $\mu \mathrm{Ci} / \mathrm{g}$ \\
\hline${ }^{242} \mathrm{Pu}$ & ICP/MS & 0.2 & 0.6 & $\mu \mathrm{Ci} / \mathrm{g}$ \\
\hline${ }^{242} \mathrm{Pu}$ & Sep./AEA & $2.0 \mathrm{E}-02$ & $6.0 \mathrm{E}-02$ & $\mu \mathrm{Ci} / \mathrm{g}$ \\
\hline${ }^{243} \mathrm{Am}$ & ICP/MS & 2 & 6 & $\mu \mathrm{Ci} / \mathrm{g}$ \\
\hline${ }^{243+244} \mathrm{Cm}$ & Sep./AEA & $4.0 \mathrm{E}-03(2.0 \mathrm{E}-05)$ & $1.2 \mathrm{E}-02(6.0 \mathrm{E}-05)$ & $\mu \mathrm{Ci} / \mathrm{g}$ \\
\hline Total alpha & Alpha counting & $2.0 \mathrm{E}-01$ & $6.0 \mathrm{E}-01$ & $\mu \mathrm{Ci} / \mathrm{g}$ \\
\hline Total beta & Liquid scint. & $7.0 \mathrm{E}+00$ & $2.1 \mathrm{E}+01$ & $\mu \mathrm{Ci} / \mathrm{g}$ \\
\hline
\end{tabular}

Notes:

AEA $=$ alpha energy analysis

CVAA $=$ cold vapor atomic absorption 
GEA = gamma energy analysis

$\mathrm{IC}=$ ion chromatography

ICP/AES = inductively coupled plasma/atomic emission spectroscopy

ICP/MS = inductively coupled plasma/mass spectrometry

ISE = ion-specific electrode

Liquid scint. $=$ liquid scintillation

Sep. $=$ separation

1 Detection limits and MRQ's for the L\&H DQO. Where HLW DQO requirements differ from those listed, the corresponding HLW requirement is shown in parentheses. The HLW requirements apply only if testing shows that dilution is not needed to meet waste transfer requirements. If process testing determines that dilution is required for transfer, requirements for analysis of the diluted waste will be as per the test plan.

2 NS, not stated for the L\&H DQO. In the event that testing shows that dilution is not required to meet waste transfer requirements, then the HLW MRQs apply. If process testing determines that dilution is required for transfer, requirements for analysis of the diluted waste will be as per the test plan.

3 No method currently available

4 Combined analysis of ${ }^{90} \mathrm{Sr}$ and ${ }^{90} \mathrm{Y}$

${ }^{5}$ Combined analysis of ${ }^{93 \mathrm{~m}} \mathrm{Nb}$ and ${ }^{93} \mathrm{Zr}$

6 Combined analysis of ${ }^{125} \mathrm{Sb}$ and ${ }^{125 \mathrm{~m}} \mathrm{Te}$

${ }^{7}$ Combined analysis of ${ }^{126} \mathrm{Sn},{ }^{126 m} \mathrm{Sb}$, and ${ }^{126} \mathrm{Sb}$

${ }^{8}$ Combined analysis of ${ }^{137} \mathrm{Cs}$ and ${ }^{137 \mathrm{~m}} \mathrm{Ba}$

${ }^{9}$ Combined analysis of ${ }^{239} \mathrm{Pu}$ and ${ }^{240} \mathrm{Pu}$

${ }^{10} \mathrm{Combined}$ analysis of ${ }^{242} \mathrm{Am},{ }^{242 m} \mathrm{Am}$, and ${ }^{242} \mathrm{Cm}$

Table 3-5. Group I Analytes (3 sheets)

\begin{tabular}{|c|c|c|}
\hline Analyte & LAW (liquids) & HLW (solids) \\
\hline $\mathrm{Al}$ & $\mathrm{X}$ & \\
\hline As & & $X$ \\
\hline B & & $\mathrm{X}$ \\
\hline $\mathrm{Ba}$ & $\mathrm{X}$ & \\
\hline $\mathrm{Be}$ & & $\mathrm{X}$ \\
\hline $\mathrm{Ca}$ & $X$ & \\
\hline $\mathrm{Cd}$ & $\mathrm{X}$ & \\
\hline $\mathrm{Ce}$ & & $\mathrm{X}$ \\
\hline $\mathrm{Cl}$ & $\mathrm{X}$ & $\mathrm{X}$ \\
\hline $\mathrm{CN}^{-}$ & & $X$ \\
\hline $\mathrm{Co}$ & & $X$ \\
\hline $\mathrm{Cr}$ & $\mathrm{X}$ & \\
\hline $\mathrm{Cs}$ & & $\mathrm{X}$ \\
\hline $\mathrm{Cu}$ & & $\mathrm{X}$ \\
\hline $\mathrm{F}^{-}$ & $\mathrm{X}$ & \\
\hline $\mathrm{Fe}$ & $\mathrm{X}$ & \\
\hline $\mathrm{Hg}$ & $\mathrm{X}$ & $X$ \\
\hline $\mathrm{K}$ & $\mathrm{X}$ & \\
\hline $\mathrm{La}$ & $X$ & $\mathrm{X}$ \\
\hline $\mathrm{Li}$ & & $\mathrm{X}$ \\
\hline $\mathrm{Mn}$ & & $\mathrm{X}$ \\
\hline Mo & & $X$ \\
\hline
\end{tabular}


RPP-5617, Rev. 1

Table 3-5. Group I Analytes (3 sheets)

\begin{tabular}{|c|c|c|}
\hline Analyte & LAW (liquids) & HLW (solids) \\
\hline $\mathrm{Na}$ & $\mathrm{X}$ & \\
\hline $\mathrm{Nd}$ & & $\mathrm{X}$ \\
\hline $\mathrm{NH}_{3}$ & & $X$ \\
\hline $\mathrm{Ni}$ & $\mathrm{X}$ & \\
\hline $\mathrm{NO}_{2}^{-}$ & $\mathrm{X}$ & $\mathrm{X}$ \\
\hline $\mathrm{NO}_{3}^{-}$ & $\mathrm{X}$ & $\mathrm{X}$ \\
\hline $\mathrm{Pb}$ & $\mathrm{X}$ & \\
\hline $\mathrm{PO}_{4}^{-3}$ & $\mathrm{X}$ & \\
\hline $\operatorname{Pr}$ & & $\mathrm{X}$ \\
\hline $\mathrm{Pu}$ & & $\mathrm{X}$ \\
\hline $\mathrm{Rb}$ & & $\mathrm{X}$ \\
\hline $\mathrm{Sb}$ & & $\mathrm{X}$ \\
\hline $\mathrm{Se}$ & & $\mathrm{X}$ \\
\hline $\mathrm{SO}_{4}^{-2}$ & $\mathrm{X}$ & \\
\hline $\mathrm{Sr}$ & & $\mathrm{X}$ \\
\hline $\mathrm{Ta}$ & & $\mathrm{X}$ \\
\hline Tc & & $\mathrm{X}$ \\
\hline $\mathrm{Te}$ & & $\mathrm{X}$ \\
\hline Th & & $\mathrm{X}$ \\
\hline TIC & $\mathrm{X}$ & $\mathrm{X}$ \\
\hline $\mathrm{Tl}$ & & $\mathrm{X}$ \\
\hline TOC & $\mathrm{X}$ & $\mathrm{X}$ \\
\hline $\mathrm{U}$ & $X$ & \\
\hline $\mathrm{V}$ & & $\mathrm{X}$ \\
\hline $\mathrm{W}$ & & $\mathrm{X}$ \\
\hline $\mathrm{Y}$ & & $\bar{X}$ \\
\hline $\mathrm{Zn}$ & & $\mathrm{X}$ \\
\hline${ }^{3} \mathrm{H}$ & & $\mathrm{X}$ \\
\hline${ }^{14} \mathrm{C}$ & & $X$ \\
\hline${ }^{60} \mathrm{Co}$ & $\mathrm{X}$ & $\mathrm{X}$ \\
\hline${ }^{90} \mathrm{Sr}$ & $\mathrm{X}$ & $\mathrm{X}$ \\
\hline${ }^{99} \mathrm{Tc}$ & $\mathrm{X}$ & $\mathrm{X}$ \\
\hline${ }^{125} \mathrm{Sb}$ & & $X$ \\
\hline${ }^{126} \mathrm{Sn}$ & & $\mathrm{X}$ \\
\hline${ }^{129} \mathrm{I}$ & & $\mathrm{X}$ \\
\hline${ }^{137} \mathrm{Cs}$ & $\mathrm{X}$ & $\mathrm{X}$ \\
\hline${ }^{152} \mathrm{Eu}$ & & $\mathrm{X}$ \\
\hline${ }^{154} \mathrm{Eu}$ & $\mathrm{X}$ & $\mathrm{X}$ \\
\hline${ }^{155} \mathrm{Eu}$ & $\mathrm{X}$ & $\mathrm{X}$ \\
\hline${ }^{233} \mathrm{U}$ & & $\mathrm{X}$ \\
\hline${ }^{235} \mathrm{U}$ & & $\mathrm{X}$ \\
\hline${ }^{237} \mathrm{~Np}$ & $\mathrm{X}$ & $\mathrm{X}$ \\
\hline
\end{tabular}




\section{RPP-5617, Rev. 1}

Table 3-5. Group I Analytes (3 sheets)

\begin{tabular}{|c|c|c|}
\hline Analyte & LAW (liquids) & HLW (solids) \\
\hline${ }^{238} \mathrm{Pu}$ & $\mathrm{X}$ & $\mathrm{X}$ \\
\hline${ }^{239} \mathrm{Pu}$ & $\mathrm{X}$ & $\mathrm{X}$ \\
\hline${ }^{240} \mathrm{Pu}$ & $\mathrm{X}$ & \\
\hline${ }^{24 !} \mathrm{Pu}$ & $\mathrm{X}$ & $\mathrm{X}$ \\
\hline${ }^{242} \mathrm{Pu}$ & $\mathrm{X}$ & \\
\hline${ }^{241} \mathrm{Am}$ & $\mathrm{X}$ & $\mathrm{X}$ \\
\hline${ }^{242} \mathrm{Cm}$ & $\mathrm{X}$ & \\
\hline${ }^{243+244} \mathrm{Cm}$ & $\mathrm{X}$ & $\mathrm{X}$ \\
\hline${ }^{243} \mathrm{Am}$ & $\mathrm{X}$ & \\
\hline
\end{tabular}




\subsection{QUALITY ASSURANCE AND QUALITY CONTROL}

Processes, services, activities, and conditions adverse to the quality which do not conform to requirements specified in this SAP or references herein shall be controlled to prevent inadvertent use. Nonconforming sampling and analysis processes shall be identified, controlled, reported, and the disposition taken as required by the Nonconforming Item Reporting and Control (CHG 1999).

\subsection{LABORATORY OPERATIONS}

Laboratories performing analyses in support of this SAP shall have approved and implemented Quality Assurance (QA) Plans. These QA plans shall meet the Hanford Analytical Services Quality Assurance Requirements Document (DOE-RL 1998) minimum requirements as the baseline for laboratory quality systems. The 222-S Laboratory Quality Assurance Plan (Markel 1999) specifies the requirements for assuring the quality of sample analysis conducted at the 222-S Laboratory. Quality requirements for conducting Characterization Project sampling and analysis are described in Tank Waste Remediation System Characterization Project, Quality Policies (Board 1998) and this SAP. Characterization Project sampling and analysis shall be conducted in conformance with these requirements.

Analytical quality control (QC) requirements (duplicates, spikes, blanks, laboratory control samples) are identified in Tables 3-1,3-2, 4-1, and 4-2. The laboratory shall also use calibration and calibration check standards appropriate for the analytical instrumentation being used (see DOE-RL [1998] for definitions of QC samples and standards). The criteria presented are goals for demonstrating reliable method performance. It is understood that the laboratory will follow its internal QC system for required actions whenever QC failures occur. If sample QC failures occur, or if all analyses cannot be performed (e.g., insufficient sample), analysts shall consult with supervisors/customers to determine the proper action. The laboratory should provide a suggested course of action at that time. All sample QC failures and limitations on the associated data shall be discussed in the narrative of the data report. Proper notification of all data not meeting QC requirements shall be included with the data.

Archive all unused segment waste material. Ensure that there is sufficient segment material so (1) a segment chemical analysis can be performed, or (2) composites based on expected retrieval batches can be prepared, at a later date. 


\subsection{SAMPLE COLLECTION}

Before sampling can be performed on a tank, available risers must be identified for use in the sampling event. The selected risers must be inspected and prepared to confirm their ability to be used in sampling. Safety hazards must be identified and special precautions must be taken if needed. If deemed necessary by the sampling cognizant engineers and tank coordinator, video surveillance should be performed to identify any potential problems that may occur during the sampling event.

Samples are to be taken from a tank and shipped to the performing laboratory by CPO in accordance with the respective work packages. The chain-of-custody forms for these work packages shall identify samples by a unique number and state the type of sampler used (retained gas sampler or universal sampler) for each sample before being shipped to the 222-S Laboratory. Approved procedure TO-080-090 [Load/Transport Sample Cask(s)] is to be used during the sampling event. Pertinent sampling information (e.g., unusual waste characteristics, X-ray scan results, $\mathrm{LiBr}$ solution used, or detecting debris) should be noted in the comment section of the chain-of-custody form.

Characterization Project Operations should transport each sample collected to the performing laboratory within three calendar days of removing the sample from the tank. A verbal notification by CPO is to be made to the 222-S Laboratory at 373-2435 at least 24 hours in advance of an expected shipment. 
RPP-5617, Rev. 1

Table 4-1. Quality Control Parameters for Liquid Analysis (2 sheets)

\begin{tabular}{|c|c|c|c|c|}
\hline \multirow[b]{2}{*}{ Analyte } & \multirow[b]{2}{*}{$\begin{array}{l}\text { Analytical } \\
\text { Technique }\end{array}$} & \multicolumn{3}{|c|}{ QC Acceptance Criteria } \\
\hline & & $\begin{array}{c}\text { LCS } \\
\text { \%Recovery }\end{array}$ & $\begin{array}{c}\text { Spike } \\
\text { \%Recovery }\end{array}$ & Triplicate RSD ${ }^{1}$ \\
\hline Separable organic & Visual & N/A & N/A & $\mathrm{N} / \mathrm{A}$ \\
\hline Specific gravity & Gravimetry & N/A & N/A & $\mathrm{N} / \mathrm{A}$ \\
\hline $\mathrm{Wt} \%$ dissolved solids & Gravimetry & $80-120 \%$ & N/A & $<21 \%$ \\
\hline $\mathrm{Wt} \%$ oxide & Gravimetry & TBD & TBD & TBD \\
\hline $\begin{array}{l}\mathrm{Ag}, \mathrm{Al}, \mathrm{As}, \mathrm{B}, \mathrm{Be}, \mathrm{Ba}, \mathrm{Bi} \\
\mathrm{Ca}, \mathrm{Cd}, \mathrm{Ce}, \mathrm{Cr}, \mathrm{Cu}, \mathrm{Eu} \\
\mathrm{Fe}, \mathrm{K}, \mathrm{La}, \mathrm{Li}, \mathrm{Mg}, \mathrm{Mn}, \\
\mathrm{Mo}, \mathrm{Nd}, \mathrm{Ni}, \mathrm{P}, \mathrm{Pb}, \mathrm{S}, \mathrm{Sb} \\
\mathrm{Se}, \mathrm{Sr}, \mathrm{Si}, \mathrm{Th}, \mathrm{Ti}, \mathrm{Tl}, \mathrm{U}, \mathrm{V}, \\
\mathrm{Zn}, \mathrm{Zr}\end{array}$ & ICP/AES & $80-120 \%$ & $75-125 \%$ & $<15 \%$ \\
\hline $\mathrm{Na}$ & ICP/AES & $80-120 \%$ & $75-125 \%$ & $<3.5 \%$ \\
\hline $\begin{array}{l}\mathrm{As}, \mathrm{B}, \mathrm{Ba}, \mathrm{Be}, \mathrm{Ce}, \mathrm{Co}, \mathrm{La}, \\
\mathrm{Li}, \mathrm{Mo}, \mathrm{Pd}, \mathrm{Pr}, \mathrm{Rb}, \mathrm{Rh}, \\
\mathrm{Ru}, \mathrm{Sb}, \mathrm{Se}, \mathrm{Ta}, \mathrm{Te}, \mathrm{Th}, \mathrm{Tl} \\
\mathrm{V}, \mathrm{W},{ }^{90} \mathrm{AMU},{ }^{162} \mathrm{Sn},{ }^{238} \mathrm{U}, \\
{ }^{241} \mathrm{AMU},{ }^{242} \mathrm{AMU},{ }^{99} \mathrm{Tc}\end{array}$ & ICP/MS & $80-120 \%$ & $70-130 \%$ & $<15 \%$ \\
\hline $\mathrm{Cs}, \mathrm{Eu}$ & ICP/MS & $\mathrm{N} / \mathrm{A}$ & $\mathrm{N} / \mathrm{A}$ & N/A \\
\hline${ }^{231} \mathrm{~Pa}$ & ICP/MS & TBD & TBD & TBD \\
\hline${ }^{233} \mathrm{U},{ }^{234} \mathrm{U},{ }^{235} \mathrm{U},{ }^{236} \mathrm{U}$, & ICP/MS & $90-110 \%$ & $75-125 \%$ & $<15 \%$ \\
\hline $\begin{array}{l}\mathrm{Br}^{-}, \mathrm{Cl}^{-}, \mathrm{F}^{-}, \mathrm{NO}_{2}, \mathrm{NO}_{3}{ }^{\prime} \\
\mathrm{PO}_{4}^{-3}, \mathrm{SO}_{4}^{-2}, \text { formate, } \\
\text { oxalate, acetate, citrate, } \\
\text { INA }\end{array}$ & IC & $80-120 \%$ & $75-125 \%$ & $<15 \%$ \\
\hline $\mathrm{Hg}$ & CVAA & $80-120 \%$ & $75-125 \%$ & $<15 \%$ \\
\hline $\mathrm{Sb}, \mathrm{Se}$ & GFAA & $80-120 \%$ & $75-125 \%$ & $<15 \%$ \\
\hline $\mathrm{NH}_{3} / \mathrm{NH}_{4}^{+}$ & $\begin{array}{l}\text { ISE, standard } \\
\text { additions }\end{array}$ & $80-120 \%$ & $75-125 \%$ & $<15 \%$ \\
\hline $\mathrm{OH}-$ & $\begin{array}{l}\text { Potentiometric } \\
\text { titration }\end{array}$ & $80-120 \%$ & N/A & $<15 \%$ \\
\hline TIC, TOC & $\begin{array}{l}\text { Persulfate and } \\
\text { furnace oxidation }\end{array}$ & $80-120 \%$ & $75-125 \%$ & $<15 \%$ \\
\hline $\mathrm{CN}^{-}$ & $\begin{array}{l}\text { Distillation/ } \\
\text { colorimetric }\end{array}$ & $80-120 \%$ & $75-125 \%$ & $<15 \%$ \\
\hline Total alpha & $\begin{array}{l}\text { Proportional } \\
\text { counting }\end{array}$ & $70-130 \%$ & $75-125 \%$ & $<15 \%$ \\
\hline Total beta & Beta counting & $70-130 \%$ & $70-130 \%$ & $<15 \%$ \\
\hline
\end{tabular}


RPP-5617, Rev. 1

Table 4-1. Quality Control Parameters for Liquid Analysis (2 sheets)

\begin{tabular}{|c|c|c|c|c|}
\hline \multirow[b]{2}{*}{ Analyte } & \multirow[b]{2}{*}{$\begin{array}{l}\text { Analytical } \\
\text { Technique }\end{array}$} & \multicolumn{3}{|c|}{ QC Acceptance Criteria } \\
\hline & & $\begin{array}{c}\text { LCS } \\
\text { \%Recovery }\end{array}$ & $\begin{array}{c}\text { Spike } \\
\% \text { Recovery }\end{array}$ & Triplicate RSD ${ }^{1}$ \\
\hline${ }^{90} \mathrm{Sr}$ & $\begin{array}{l}\text { Separation/beta } \\
\text { counting }\end{array}$ & $75-125 \%$ & N/A & $<15 \%$ \\
\hline $\begin{array}{l}{ }^{238} \mathrm{Pu},{ }^{239 / 240} \mathrm{Pu},{ }^{241} \mathrm{Am}, \\
{ }^{242} \mathrm{Cm},{ }^{243 / 244} \mathrm{Cm}\end{array}$ & Separation/AEA & NP & N/A & $<15 \%$ \\
\hline${ }^{3} \mathrm{H}$ & $\begin{array}{l}\text { Separation/liquid } \\
\text { scintillation }\end{array}$ & $80-120 \%$ & N/A & $<15 \%$ \\
\hline${ }^{14} \mathrm{C}$ & $\begin{array}{l}\text { Separation/liquid } \\
\text { scintillation }\end{array}$ & $80-120 \%$ & $75-125 \%$ & $<15 \%$ \\
\hline${ }^{79} \mathrm{Se}$ & Liquid scintillation & NP & N/A & $<15 \%$ \\
\hline${ }^{99} \mathrm{Tc}$ & ICP/MS & $80-120 \%$ & $70-130 \%$ & $<15 \%$ \\
\hline${ }^{99} \mathrm{Tc},{ }^{99} \mathrm{Tc}$ (pertechnetate) & $\begin{array}{l}\text { Separation/beta } \\
\text { count }\end{array}$ & $80-120 \%$ & $70-130 \%$ & $<15 \%$ \\
\hline${ }^{60} \mathrm{Co},{ }^{157} \mathrm{Cs},{ }^{152} \mathrm{Eu},{ }^{154} \mathrm{Eu}$, & GEA & NP & N/A & $<15 \%$ \\
\hline${ }^{125} \mathrm{Sb}$ & GEA & TBD & TBD & TBD \\
\hline${ }^{129} \mathrm{I}$ & Separation/GEA & NP & $\mathrm{N} / \mathrm{A}$ & $<15 \%$ \\
\hline
\end{tabular}

Notes:

$\mathrm{AEA}=$ alpha energy analysis

$\mathrm{AMU}=$ atomic mass unit

$\mathrm{CVAA}=$ cold vapor atomic absorption

GEA = gamma energy analysis

GFAA $=$ graphite furnace atomic absorption

$\mathrm{IC}=$ ion chromatography

$\mathrm{ICP} / \mathrm{MS}=$ inductively coupled plasma/mass spectrometry

ICP/AES = inductively coupled plasma/atomic emission spectroscopy

ISE $=$ ion specific electrode

LCS $=$ laboratory control standard

$\mathrm{N} / \mathrm{A}=$ not applicable

$\mathrm{NP}=$ not performed

$\mathrm{TBD}=$ to be determined

$\mathrm{TIC}=$ total inorganic carbon

TOC $=$ total organic carbon

RSD $=$ relative standard deviation

$\mathrm{Wt} \%=$ weight percent

'RSD requirement if the sample result is at least 10 times the instrument detection limit. RSD $=$ (standard deviation of the mean/mean) $\times 100 \%$ 
Table 4-2. Quality Control Parameters for Solids Analysis (2 Sheets)

\begin{tabular}{|c|c|c|c|c|}
\hline \multirow[b]{2}{*}{ Solids Fraction } & \multirow[b]{2}{*}{ Analytical Technique } & \multicolumn{3}{|c|}{ QC Acceptance Criteria } \\
\hline & & $\begin{array}{c}\text { LCS \% } \\
\text { Recovery }\end{array}$ & $\begin{array}{l}\text { Spike \% } \\
\text { Recovery }\end{array}$ & $\begin{array}{l}\text { Triplicate } \\
\text { RSD }^{1}\end{array}$ \\
\hline Shear strength & Rotary viscometer & NS & N/A & N/A \\
\hline Particle size distribution & Particle size analyzer & N/A & N/A & N/A \\
\hline Bulk density & Centrifugation & N/A & N/A & N/A \\
\hline $\mathrm{Wt} \%$ solids & Gravimetry & $80-120 \%$ & N/A & $<21 \%$ \\
\hline $\mathrm{Wt} \%$ oxide & Gravimetry & TBD & TBD & TBD \\
\hline $\begin{array}{l}\mathrm{Ag}, \mathrm{Al}, \mathrm{As}, \mathrm{B}, \mathrm{Ba}, \mathrm{Be}, \\
\mathrm{Bi}, \mathrm{Ca}, \mathrm{Cd}, \mathrm{Ce}, \mathrm{Cr}, \mathrm{Cu}, \\
\mathrm{Fe}, \mathrm{K}, \mathrm{La}, \mathrm{Mg}, \mathrm{Mn}, \mathrm{Li} \\
\mathrm{Nd}, \mathrm{Ni}, \mathrm{P}, \mathrm{Pb}, \mathrm{S}, \mathrm{Sb}, \mathrm{Se} \text {, } \\
\mathrm{Si}, \mathrm{Sr}, \mathrm{Th}, \mathrm{Ti}, \mathrm{Tl}, \mathrm{U}, \mathrm{V}, \\
\text { Y, Zn, Zr }\end{array}$ & ICP/AES & $80-120 \%$ & $75-125 \%$ & $<15 \%$ \\
\hline $\mathrm{Na}$ & ICP/AES & $80-120 \%$ & $75-125 \%$ & $<3.5 \%$ \\
\hline $\begin{array}{l}\text { Ag, As, B, Be, Ce, Co, K, } \\
\text { La, Li, Mo, Nd, Pd, Pr, } \\
\text { Rb, Rh, Ru, S, Sb, Se, } \\
\text { Ta, Te, Th, Tl, V, W, Y, } \\
\text { Zn, }{ }^{90} \text { AMU }\end{array}$ & ICP/MS & $80-120 \%$ & $70-130 \%$ & $<15 \%$ \\
\hline $\mathrm{Cs}$ & ICP/MS & N/A & N/A & N/A \\
\hline${ }^{233} \mathrm{U},{ }^{234} \mathrm{U},{ }^{237} \mathrm{U},{ }^{236} \mathrm{U}$, & ICP/MS & $90-110 \%$ & $75-125 \%$ & $<15 \%$ \\
\hline $\begin{array}{l}{ }^{99} \mathrm{Tc},{ }^{126} \mathrm{Sn},{ }^{135} \mathrm{Cs}, \\
{ }^{151} \mathrm{AMU},{ }^{238} \mathrm{U},{ }^{241} \mathrm{AMU}, \\
{ }^{242} \mathrm{AMU}\end{array}$ & ICP/MS & $80-120 \%$ & $70-130 \%$ & $<15 \%$ \\
\hline${ }^{63} \mathrm{Ni}$ & TBD & TBD & N/A & $<15 \%$ \\
\hline${ }^{93} \mathrm{AMU}$ & TBD & TBD & N/A & $<15 \%$ \\
\hline${ }^{121 \mathrm{~m}} \mathrm{Sn}$ & TBD & TBD & N/A & $<15 \%$ \\
\hline $\begin{array}{l}\mathrm{Br}, \mathrm{Cl}^{-}, \mathrm{F}^{-}, \mathrm{NO}_{2}^{-}, \mathrm{NO}_{3}{ }^{-}, \\
\mathrm{PO}_{4}^{-3}, \mathrm{SO}_{4}^{-2}, \text { formate, } \\
\text { oxalate }\end{array}$ & IC & $80-120 \%$ & $75-125 \%$ & $<15 \%$ \\
\hline $\mathrm{Hg}$ & CVAA & $80-120 \%$ & $75-125 \%$ & $<15 \%$ \\
\hline $\mathrm{Sb}, \mathrm{Se}$ & GFAA & $80-120 \%$ & $75-125 \%$ & $<15 \%$ \\
\hline $\mathrm{OH}$ & Potentiometric titration & $80-120 \%$ & $\mathrm{~N} / \mathrm{A}$ & $<15 \%$ \\
\hline $\mathrm{NH}_{3} / \mathrm{NH}_{4}{ }^{+}$ & ISE, standard additions & $80-120 \%$ & $75-125 \%$ & $<15 \%$ \\
\hline TIC, TOC & $\begin{array}{l}\text { Persulfate and furnace } \\
\text { oxidation }\end{array}$ & $80-120 \%$ & $75-125 \%$ & $<15 \%$ \\
\hline $\mathrm{CN}^{-}$ & $\begin{array}{l}\begin{array}{l}\text { Distillation/ } \\
\text { colorimetric }\end{array} \\
\end{array}$ & $80-120 \%$ & $75-125 \%$ & $<15 \%$ \\
\hline Total Alpha & Proportional counter & $70-130 \%$ & $75-125 \%$ & $<15 \%$ \\
\hline Total Beta & Beta counting & $70-130 \%$ & $70-130 \%$ & $<15 \%$ \\
\hline
\end{tabular}


RPP-5617, Rev. 1

Table 4-2. Quality Control Parameters for Solids Analysis (2 Sheets)

\begin{tabular}{|c|c|c|c|c|}
\hline \multirow[b]{2}{*}{ Solids Fraction } & \multirow[b]{2}{*}{ Analytical Technique } & \multicolumn{3}{|c|}{ QC Acceptance Criteria } \\
\hline & & $\begin{array}{l}\text { LCS \% } \\
\text { Recovery }\end{array}$ & $\begin{array}{l}\text { Spike \% } \\
\text { Recovery }\end{array}$ & $\begin{array}{c}\text { Triplicate } \\
\text { RSD }^{1}\end{array}$ \\
\hline${ }^{90} \mathrm{Sr}$ & $\begin{array}{l}\text { Isotopic specific } \\
\text { separation/beta } \\
\text { counting }\end{array}$ & $75-125 \%$ & N/A & $<15 \%$ \\
\hline${ }^{238} \mathrm{Pu},{ }^{239 / 240} \mathrm{Pu}$ & Separation/AEA & NP & $70-130 \%$ & $<15 \%$ \\
\hline${ }^{241} \mathrm{Am}$ & Separation/AEA & $80-120 \%$ & N/A & $<15 \%$ \\
\hline${ }^{242} \mathrm{Cm},{ }^{243 / 244} \mathrm{Cm}$ & Separation/AEA & $\mathrm{NP}$ & $\mathrm{N} / \mathrm{A}$ & $<15 \%$ \\
\hline${ }^{3} \mathrm{H}$ & $\begin{array}{l}\text { Separation/liq. } \\
\text { Scintillation }\end{array}$ & $80-120 \%$ & $\mathrm{~N} / \mathrm{A}$ & $<15 \%$ \\
\hline${ }^{14} \mathrm{C}$ & $\begin{array}{l}\text { Separation/liquid } \\
\text { scintillation }\end{array}$ & $80-120 \%$ & $75-125 \%$ & $<15 \%$ \\
\hline${ }^{99} \mathrm{Tc}$ & $\begin{array}{l}\text { Separation/liquid } \\
\text { scintillation }\end{array}$ & $80-120 \%$ & $70-130 \%$ & $<15 \%$ \\
\hline${ }^{60} \mathrm{Co}$ & GEA & $80-120 \%$ & $\mathrm{~N} / \mathrm{A}$ & $<15 \%$ \\
\hline $\begin{array}{l}{ }^{125} \mathrm{Cs},{ }^{152} \mathrm{Eu},{ }^{154} \mathrm{Eu},{ }^{155} \mathrm{Eu}, \\
{ }^{15}\end{array}$ & GEA & $\mathrm{NP}$ & N/A & $<15 \%$ \\
\hline${ }^{129} \mathrm{I}$ & Separation/GEA & NP & N/A & $<15 \%$ \\
\hline${ }^{59} \mathrm{Ni}$ & Separation/GEA & $\mathrm{NP}$ & N/A & $<15 \%$ \\
\hline${ }^{126} \mathrm{Sb},{ }^{126 \mathrm{~m}} \mathrm{Sb}$ & Separation/GEA & NP & N/A & $<15 \%$ \\
\hline
\end{tabular}

Notes:

AEA $=$ alpha energy analysis

CVAA $=$ cold vapor atomic absorption

$\mathrm{GEA}=$ gamma energy analysis

GFAA = graphite furnace atomic absorption

$\mathrm{IC}=$ ion chromatography

ICP/AES = inductively coupled plasma/atomic emission spectroscopy

ICP/MS = inductively coupled plasma/mass spectrometry

ISE $=$ ion specific electrode

LCS = laboratory control standard

$\mathrm{N} / \mathrm{A}=$ not applicable

$\mathrm{NP}=$ not performed

$\mathrm{RSD}=$ relative Standard Deviation

$\mathrm{TBD}=$ to be determined

$\mathrm{TIC}=$ total inorganic carbon

$\mathrm{TOC}=$ total organic carbon

$\mathrm{Wt} \%=$ weight percent

${ }^{1} \mathrm{RSD}$ requirement if the sample result is at least 10 times the instrument detection limit. RSD $=$ (standard deviation of the mean/mean) $\times 100 \%$ 


\subsection{SAMPLE CUSTODY}

The chain-of-custody form is initiated by the sampling team as described in the work packages. Samples are shipped in a cask and sealed with a Waste Tank Sample Seal (see below).

\begin{tabular}{||l|l||}
\hline \multicolumn{2}{|c|}{ WASTE TANK SAMPLE SEAL } \\
\hline Supervisor: & Sample No.: \\
\hline Date of Sampling: & Time of Sampling: \\
\hline Shipment No.: & Serial No.: \\
\hline
\end{tabular}

Each sample number shall be created using the sample's core and segment number. For instance, segment 1 of core 197 would be sample number 197-01. The sealed and labeled samples are shipped to the laboratory along with the chain-of-custody form. The receipt and control of samples in the 222-S Laboratory are described in laboratory procedure LO-090-101. 
RPP-5617, Rev. 1

\subsection{EXCEPTIONS, CLARIFICATIONS, AND ASSUMPTIONS}

\subsection{EXCEPTIONS TO DATA QUALITY OBJECTIVES REQUIREMENTS}

Baldwin (1999) requires additional analyses be performed in support of the HLW DQO. In addition to the analyses performed on core composites to support the HLW DQO, analyses on the bottom two half-segments of sludge are required. Viscosity and settling rate measurements in support of the LAW and HLW DQOs are not within the scope of this SAP and will be addressed in a test plan to be prepared by the Process Chemistry Laboratory. Particle size distribution will be measured on at least one subsample from each solids layer in tank 241-AY-101.

For the Equipment DQO (Bloom 1996), only shear strength is required.

The L\&H DQO requires full dissolution of solids samples for analysis. However, some of the analyses in Table 3-2 can only be performed on water digests of solids; full dissolution of the sample for analysis is not currently possible for these analytes. These analytes are: TIC and TOC by furnace oxidation, anions by IC, tritium, carbon-14, and iodine- 129 .

Many of the analyses performed in triplicate as directed by the L\&H DQO are also required in duplicate by the HLW and LAW DQOs. Per customer request, duplication of effort is to be avoided by performing these analyses in triplicate as directed by the L\&H DQO to satisfy the requirements of the HLW and LAW DQOs in the event that dilution is not required. Tables 3-1 through 4-2 reflect compromises between these DQO requirements to satisfy the affected programs. Several analytes being measured by ICP-MS required by the L\&H DQO will take the place of ICP-AES analyses for liquids (Ba and $\mathrm{La}$ ) and solids (As, B, Be, Ce, Co, Cs, Li, Mo, Pr, $\mathrm{Rb}, \mathrm{Sb}, \mathrm{Se}, \mathrm{Ta}, \mathrm{Te}, \mathrm{Th}, \mathrm{Tl}, \mathrm{V})$ required by the HLW and LAW DQOs.

The tables identify many analytes to be determined by ICP/MS because this technique has the sensitivity to meet the desired MRQs. However, if the concentration of the analyte is high enough the quality of the ICP/AES results will be as good as, or better than, the ICP/MS data. Because ICP/MS is based on the measurement of different element isotopes, the total amount of an element must be determined by summing the isotopes or by calculations assuming the natural abundance of the isotopes. However, in the production of nuclear materials these natural abundances are changed, particularly around the mass peaks of the fission product yield curves (AMU-90 and -137). The presence of the fission products in the samples can lead to unnatural isobaric interference that can lead to inaccuracies in the measurements. Another potential interference to the ICP/MS method is in the low ( $<80 \mathrm{AMU})$ atomic mass range where polyatomic species and ionized species from the argon plasma gas can cause interference problems. Elements in this region may be determined easier and more accurately using ICP/AES if the concentrations in the sample are high enough. Because it is not possible to precisely predict what trace analytes will be present in high enough concentrations for ICP/AES analysis, ICP/AES and ICP/MS are being requested for all possible analytes. For liquid samples, the following elements cannot be analyzed by ICP/AES at the required MRQs: As, B, Ba, Be (borderline), $\mathrm{Ce}, \mathrm{Co}, \mathrm{K}, \mathrm{La}, \mathrm{Li}, \mathrm{Mo}, \mathrm{Sb}, \mathrm{Se}, \mathrm{Th}, \mathrm{Tl}$, and V. For solid samples the following 
elements cannot be analyzed by ICP/AES at the required MRQs: Ag (borderline), As, B, Ce, Co, La (borderline), Li, Mo, Na (borderline), Nd (borderline), S (borderline), Sb, Se, Th, Tl, U (borderline), V, Y, and $\mathrm{Zn}$ (borderline). The following analytes are expected to be semiquantitative for the ICP/MS method: Ag, Ba, Mo, Nd, Pd, Rb, Ru, Sb, Se, Te, and ${ }^{231} \mathrm{~Pa}$. Sb and Se can be performed by graphite furnace atomic absorption (GFAA) and are, therefore, requested by that method.

The 222-S laboratory does not have analytical methods for ${ }^{59} \mathrm{Ni},{ }^{63} \mathrm{Ni},{ }^{126} \mathrm{Sb},{ }^{126 \mathrm{~m}} \mathrm{Sb},{ }^{121 \mathrm{~m}} \mathrm{Sn},{ }^{93} \mathrm{Nb}$, or ${ }^{93} \mathrm{Zr}$.

\subsection{CLARIFICATIONS AND ASSUMPTIONS}

The laboratory is requested to report all analytical results recovered from multi-analyte methods, including the inductively coupled plasma - atomic emission spectroscopy (ICP/AES), gamma energy analysis (GEA), and ion chromatography (IC) analyses, even though only specific analytes are requested. These opportunistic analyses (Kristofzski 1996) should be reported only if no additional preparatory work is required (e.g., running additional standards) and if the associated QC results (blanks, standards, and spikes, as appropriate) are reported. No reruns or additional analyses should be performed to improve recovery for analytes not specifically requested in Tables 3-1 or 3-2. 


\subsection{ORGANIZATION}

The organization and responsibility of points of contact and key personnel involved with this tank 241-AY-101 characterization project are listed in Table 6-1.

Table 6-1. Tank 241-AY-101 Project Points of Contact and Key Personnel List

\begin{tabular}{|c|c|c|}
\hline Responsibility & Organization & Individual \\
\hline $\begin{array}{l}\text { RPP 241-AY-101 Tank } \\
\text { Coordinator }\end{array}$ & $\begin{array}{l}\text { RPP Process Engineering } \\
(\mathrm{CHG})\end{array}$ & A. M. Templeton, 373-5589 \\
\hline $\begin{array}{l}\text { 222-S Project Coordinator for } \\
\text { 241-AY-101 }\end{array}$ & Analytical Production $(\mathrm{FH})$ & K. E. Bell, 372-2553 \\
\hline $\begin{array}{l}\text { 222-S Laboratory Point of } \\
\text { Contact (day shift) }\end{array}$ & Analytical Services (FH) & W. I. Winters, 373-1951 \\
\hline $\begin{array}{l}\text { 222-S Laboratory Point of } \\
\text { Contact }\end{array}$ & Analytical Services (FH) & $\begin{array}{l}\text { 222-S Laboratory Shift } \\
\text { Manager, 373-2435 }\end{array}$ \\
\hline $\begin{array}{l}200 \text { East Tank Farm Point of } \\
\text { Contact }\end{array}$ & Tank Farm Operations (CHG) & $\begin{array}{l}\text { East Tank Farm Operations } \\
\text { Shift Manager, } 373-3475\end{array}$ \\
\hline Data Management & $\begin{array}{l}\text { Data Development and } \\
\text { Interpretation (CHG) }\end{array}$ & J. G. Field, 376-3753 \\
\hline $\begin{array}{l}\text { Process Engineering Point of } \\
\text { Contact for Immediate } \\
\text { Notifications }\end{array}$ & $\begin{array}{l}\text { RPP Process Engineering } \\
(\mathrm{CHG})\end{array}$ & $\begin{array}{l}\text { On-Call Process Engineer, } \\
539-2074 \text { or } 85-9654 \text { (pager) }\end{array}$ \\
\hline $\begin{array}{l}\text { Process Chemistry Point of } \\
\text { Contact }\end{array}$ & $\begin{array}{l}\text { Manager, Technology, } \\
\text { Operations and Process } \\
\text { Science (NHC) }\end{array}$ & L. L. Lockrem, 373-4471 \\
\hline WFD Point of Contact & $\begin{array}{l}\text { Retrieval Engineering } \\
(\mathrm{NHC} / \mathrm{CHG})\end{array}$ & J. H. Baldwin, 373-4533 \\
\hline $\begin{array}{l}\text { WIT Technical Point of } \\
\text { Contact }\end{array}$ & $\begin{array}{l}\text { Pacific Northwest National } \\
\text { Laboratory (PNNL) }\end{array}$ & I. E. Burgeson, 372-3650 \\
\hline $\begin{array}{l}\text { Characterization Point of } \\
\text { Contact }\end{array}$ & $\begin{array}{l}\text { Characterization Project } \\
\text { Operations (CPO) }\end{array}$ & J. S. Lee, 373-0258 \\
\hline
\end{tabular}




\subsection{DELIVERABLES}

All analyses will be reported as Formats I or IV as indicated in Tables 3-1 and 3-2. Additional information regarding reporting formats is given in Schreiber (1998).

\subsection{FORMAT I REPORTING}

Tables 3-1 and 3-2 contain the notification limits for each analyte. Any results exceeding their notification limits shall be reported via telephone by the 222-S Laboratory Facility Planning Team to the East Tank Farm Operations shift manager as soon as the data are obtained and reviewed by the responsible scientist. This verbal notification must be followed within one hour by electronic notification to the tank farm operations shift manager, the River Protection Project (RPP) Process Engineering Data Development and Interpretation manager, the On-Call Process Engineer, and the tank coordinator responsible for the tank. Additional analyses for verification purposes may be contracted between the performing laboratory and the tank coordinator by either a revision to this $\mathrm{SAP}$ or by a letter.

\subsection{FORMAT IV REPORTING}

The format IV report shall be a data package reporting the results of analyses performed and will resemble a regulatory data package without third party validation. The data package should be prepared by tank and include the data for all samples, including (as applicable) formation of composites, segments, sub-segments, drainable liquids, and associated blanks taken and analyzed for this sampling event. The recommended reporting format and the raw data that shall be included are given in Section A5.0 of Schreiber (1998). The data package shall be issued 180 days after the last sample is received at the laboratory. The raw data shall be accessible to the program in accordance with the laboratory's Records Inventory and Disposition Schedule and until the respective waste tank is closed or the waste is treated.

In addition to this data package, an electronic version of the analytical results shall be provided to the Tank Characterization Database representative on the same day that the final data package is issued. The data must be available to the Washington State Department of Ecology within seven (7) days of release of the data package. The electronic version shall be in the standard electronic format (Lang et al. 1999). 


\subsection{CHANGE CONTROL}

Under certain circumstances, it may become necessary for the performing laboratory to make decisions concerning a sample without review of the data by the customer or the Characterization Project. All significant changes (such as analyte additions or analysis of new, additional samples) shall be documented by RPP Process Engineering via an Engineering Change Notice to this SAP, by a letter, or on the Characterization Change Notice Form attached in Appendix C. All changes shall also be clearly documented in the final data report. Insignificant changes may be made by the tank or project coordinator by placing a notation in the permanent record (i.e., note change in the extrusion log book or memorandum to file). Significance is determined by the tank coordinator.

At the request of the Characterization Project, additional analysis of sample material from this characterization project shall be performed following a revision of this SAP or issuance of a letter. 
RPP-5617, Rev. 1

\subsection{REFERENCES}

Baldwin, J. H., 1999, Letter of Instruction for 241-AY-101 Laboratory Work, (letter 82400-99-072 to J. G. Field, November 17), Lockheed Martin Hanford Corporation, Richland, Washington.

Bloom, G. R., 1996, Characterization Data Needs for Development, Design, and Operation of Retrieval Equipment Developed through the Data Quality Objective Process, WHC-SD-WM-DQO-008, Rev. 1, Westinghouse Hanford Company, Richland, Washington.

Board, D.C., 1998, Tank Waste Remediation System, Characterization Project, Quality Policies, HNF-SD-WM-QAPP-025, Rev. 4, Lockheed Martin Hanford Corporation, Richland, Washington.

CHG, 1999, Nonconforming- Item Reporting and Control, RPP-PRO-298, Rev. 0, CH2M HILL Hanford Group, Inc., Richland, Washington.

DOE-RL, 1998, Hanford Analytical Services Quality Assurance Requirements Document, DOE/RL-96-68, Rev. 2, U.S. Department of Energy, Richland Operations Office, Richland, Washington.

Kristofzski, J. G., 1996, Directions for "Opportunistic Analyses, " (Interoffice memorandum 75310-96-168, to J. H. Baldwin et al., September 11), Westinghouse Hanford Company, Richland, Washington.

Lang, L. L., Bobrowski, S. F., and S. J. Harris, 1999, Standard Electronic Format Specification for Tank Characterization Database Loader, Version 3.0, HNF-3638, Rev. 1, prepared by Pacific Northwest National Laboratory for Lockheed Martin Hanford Corporation, Richland, Washington.

Markel, L. P., 1999, 222-S Laboratory Quality Assurance Plan, HNF-SD-CP-QAPP-016, Rev. 3C, Waste Management Hanford, Inc., Richland, Washington.

Nguyen, D. M., 1999a, Data Quality Objectives for RPP Privatization Phase 1: Confirm Tank T is an Appropriate Feed Source for High-Level Waste Feed Batch X, HNF-1558, Rev. 2, Lockheed Martin Hanford Corporation, Richland, Washington.

Nguyen, D. M., 1999b, Data Quality Objectives for TWRS Privatization Phase 1: Confirm Tank $T$ is an Appropriate Feed Source for Low-Activity Waste Feed Batch X, HNF-1796, Rev. 2, Lockheed Martin Hanford Corporation, Richland, Washington.

Patello, G. K., M. J. Truex, and K. D. Wiemers, 1999, Low-Activity Waste and High Level Waste Feed Data Quality Objectives, PNNL-12163, Pacific Northwest National Laboratory, Richland, Washington. 
RPP-5617, Rev. 1

Schreiber, R. D., 1998, Fiscal Year 1999 Memorandum of Understanding for the TWRS Characterization Project, HNF-3578, Rev. 0, Lockheed Martin Hanford Corporation, Richland, Washington.- 
RPP-5617, Rev. 1

APPENDIX A:

EXPECTED PHYSICAL PROFILE OF TANK 241-AY-101 CORE SAMPLES 
RPP-5617, Rev. 1

APPENDIX A:

EXPECTED PHYSICAL PROFILE OF TANK 241AY-101 CORE SAMPLES

Table A-1: Tank 241-AY-101 Physical Profile Estimate Risers 15G and 150

\begin{tabular}{|c|c|c|l|l|}
\hline Segment \# & Inches & $\begin{array}{c}\text { Elevation Range } \\
\text { (ft. MSL) }\end{array}$ & \multicolumn{1}{|c|}{ Waste Type } & \multicolumn{1}{c|}{ Comments } \\
\hline \multirow{2}{*}{1} & 1.0 & & Air & \\
\cline { 2 - 5 } & 18.0 & - & Liquid & Good Recovery \\
\hline 2 & 4.0 & & Liquid & Good Recovery \\
\cline { 2 - 5 } & 15.0 & - & Sludge & Good recovery \\
\hline 3 & 19.0 & - & Sludge & Good recovery \\
\hline
\end{tabular}

Note: elevations based on inside tank bottom elevation for Tank 241-AY-101 of $623.10 \mathrm{ft}$. MSL. $\mathrm{MSL}=$ Mean Sea Level 
RPP-5617, Rev. 1

APPENDIX B:

241-AY-101 WASTE COMPOSITE PREPARATION GUIDELINES

B-1 
RPP-5617, Rev. 1

\section{APPENDIX B:}

\section{1-AY-101 WASTE COMPOSITE PREPARATION GUIDELINES}

These preparation steps for the 241-AY-101 waste composite satisfies the HLW, LAW, and the L\&H DQO objective that the composite be representative of the waste to be retrieved and allows the composite to be prepared in multiple jars.

\section{Core Sample Extrusion:}

Cores shall be extruded onto the extrusion tray. Solids and drainable liquids shall be placed in separate jars. A chemist shall note the degree of separation achieved both for the solids and the drainable liquids. If the drainable liquid contains a high concentration of solids, the chemist needs to determine if centrifuging is needed to separate the solids. The composite preparation assumes minimal solids in the drainable liquids and minimal liquid in the extruded solids. Error is introduced in the composite preparation when there is a high concentration of solids in the drainable liquid because of the difficulty in keeping the solids suspended during the liquid transfer into the composite jars. When excess liquid is in the solids, again maintaining homogeneity of the transferred solids is difficult. When making a decision to centrifuge, the composite preparation errors need to be balanced with errors associated with increased sample handling and transfer associated with the centrifuging operation.

During the entire sample extrusion process and possible subsequent sample preparation, a thorough material balance needs to be maintained and unaccounted for mass loss shall be less than $10 \%$. Observations of sample jars are very important to understanding and interpretation of later analyses.

\section{Composite Preparation:}

The composite preparation steps assume that each core segment has a jar of drainable liquids and a jar of solids. If a segment has only one jar, the steps below still apply. The number of composite jars prepared is dependent on the quantity of composite needed to meet the requirements in the Sampling and Analysis Plan (SAP). This compositing procedure requires that the composite be prepared using two aliquots of solids and two aliquots of liquid from each segment that is used as part of the composite.

Use the following steps to prepare a set of whole tank composites in individual composite jars.

1. Based on the quantity of composite required, the number of segments, and distribution of segments sampling locations in the tank, determine the weight of solids required from each segment to prepare each individual composite jar (reference calculations in the L\&H DQO).

2. Determine the weight of the solids aliquot for each segment where the solids aliquot weight equals the solids weight determined above divided by two. 
3. Based on the quantity of composite required, the number of segments, and distribution of segments sampling locations in the tank, determine the weight of liquids required from each segment to prepare each of the composite jars (reference calculations in the DQO).

4. Determine the weight of the liquid aliquot for each segment where the liquid aliquot weight equals the liquid weight determined above divided by two.

5. Homogenize the contents of the first segment solids jar by mixing.

6. Distribute a solids aliquot from the homogenized segment solids jar (weight calculated above) into each individual composite jar.

7. Homogenize the contents of the first segment liquids jar by mixing.

8. Distribute a liquid aliquot from the homogenized segment liquid jar (weight calculated above) into each individual composite jar.

9. Repeat steps 5 through 8 for each segment to be used in preparing the composites. Fill the composite jars in a random order so that the fill order is changed every time that steps 5 through 8 are repeated Note that homogenization of the sample jar before transferring an aliquot is important.

The first solids aliquot and liquid aliquot from each segment should now have been transferred to each individual composite jar. The following steps will complete the composite preparation by distributing the second solids aliquot and second liquid aliquot from each segment into the individual composite jars.

10. Rehomogenize the contents of the first segment solids jar by mixing.

11. Distribute a solids aliquot from the homogenized segment solids jar (weight calculated above) into each individual composite jar.

12. Rehomogenize the contents of the first segment liquids jar by mixing.

13. Distribute a liquid aliquot from the homogenized segment liquid jar (weight calculated above) into each individual composite jar.

14. Repeat steps 10 through 13 for each segment to be used in preparing the composites. Fill the composite jars in a random order so that the fill order is changed every time that steps 10 through 13 are repeated. Note that homogenization of the sample jar before transferring an aliquot is important.

The composite preparation is now complete. The following measurements are required for the composites.

15. Allow the composite jars to settle undisturbed for 12 to 24 hours.

16. Measure and document the volume \% settled solids for each composite jar. 
Compositing steps shall be conducted in the presence of a chemist. The attending chemist is to compare jar-to-jar variability based on observed volume \% settled solids. Any adjustments to the composite are to be documented and are to accommodate preservation of the sample pedigree to the extent possible. The attending chemist shall direct adjustments to the composite. 
RPP-5617, Rev. 1

APPENDIX C

CHARACTERIZATION CHANGE NOTICE FORM

C-1 
Document:

Change Number:

ECN to TSAP Required?

Requestor:

Date:

Samples Impacted:

Proposed Change:

Reason for Change:

Date Change Effective:

Schedule Impact:

Authorization:

Tank Coordinator:

Date:

Project Coordinator:

Date:

222-S Client Services:

Date:

Other:

Date: 


\begin{tabular}{|c|c|c|c|c|c|}
\hline \multicolumn{6}{|c|}{ DISTRIBUTION SHEET } \\
\hline \multirow[b]{2}{*}{ Distribution } & \multirow{2}{*}{\multicolumn{3}{|c|}{$\begin{array}{l}\text { From } \\
\text { Data Development ar }\end{array}$}} & \multicolumn{2}{|l|}{ Page 1 of 2} \\
\hline & & & & \multicolumn{2}{|c|}{ Date $\quad 04 / 26 / 00$} \\
\hline \multirow{2}{*}{\multicolumn{4}{|c|}{$\begin{array}{l}\text { Project Title/Work Order } \\
\text { RPP-5617, Rev. 1, "Tank 241-AY-101 Privatization Push Mode Core } \\
\text { Sampling and Analysis Plan" }\end{array}$}} & \multirow{2}{*}{\multicolumn{2}{|c|}{$\begin{array}{ll}\text { EDT No. } & \text { N/A } \\
\text { ECN No. } & E C N-657324 \\
\end{array}$}} \\
\hline & & & & & \\
\hline Name & MSIN & $\begin{array}{l}\text { Text } \\
\text { With } \\
\text { A17 } \\
\text { Attach. }\end{array}$ & Text Only & $\begin{array}{l}\text { Attach./ } \\
\text { Appendix } \\
\text { Only }\end{array}$ & $\begin{array}{l}\text { EDT/ECN } \\
\text { Only }\end{array}$ \\
\hline $\begin{array}{l}\text { CH2M HILL Hanford Group, Inc. } \\
\text { M. R. Adams } \\
\text { W. L. Adams } \\
\text { D. G. Baide } \\
\text { J. H. Baldwin } \\
\text { R. J. Brown } \\
\text { T. W. Crawford } \\
\text { R. N. Dale } \\
\text { J. G. Field } \\
\text { E. I. Husa } \\
\text { J. Jo } \\
\text { S. G. Mckinney } \\
\text { J. S. Lee } \\
\text { R. M. Orme } \\
\text { R. W. Powel1 } \\
\text { J. H. Rasmussen } \\
\text { J. F. Sickels } \\
\text { G. A. Stanton } \\
\text { G. R. Tardiff } \\
\text { A. M. Templeton } \\
\text { R. R. Thompson } \\
\text { T.C.S.R.C. }\end{array}$ & $\begin{array}{l}\text { R2 }-12 \\
\text { S6-15 } \\
\text { S5-05 } \\
\text { R3-73 } \\
\text { SO-09 } \\
\text { R3-73 } \\
\text { S7-12 } \\
\text { R2-12 } \\
\text { R2-12 } \\
\text { R2-12 } \\
\text { R2-12 } \\
\text { S7-03 } \\
\text { R3-73 } \\
\text { R3-75 } \\
\text { R2-12 } \\
\text { S7-03 } \\
\text { S7-01 } \\
\text { S5-05 } \\
\text { R2-12 } \\
\text { R2-12 } \\
\text { R1-10 }\end{array}$ & $\begin{array}{l}x \\
x \\
x \\
x \\
x \\
x \\
x \\
x \\
x \\
x \\
x \\
x \\
x \\
x \\
x \\
x \\
x \\
x \\
x \\
x \\
x \\
x \\
x\end{array}$ & & & \\
\hline $\begin{array}{l}\text { Cogema } \\
\text { T. W. Staehr } \\
\text { M. P. Rung } \\
\text { Y. S. Tran }\end{array}$ & $\begin{array}{l}\text { R3-74 } \\
\text { S7-70 } \\
\text { R3-86 }\end{array}$ & $\begin{array}{l}x \\
x \\
x\end{array}$ & & & \\
\hline $\begin{array}{l}\text { Fluor Hanford } \\
\text { R. } \text { Akita } \\
\text { K. E. Be 1l } \\
\text { L. A. Burchfield } \\
\text { R. A. Esch } \\
\text { R. K. Fuller } \\
\text { J. C. Person } \\
\text { K. L. Powe11 } \\
\text { F. H. Steen }\end{array}$ & $\begin{array}{l}\text { T6-50 } \\
\text { T6-12 } \\
\text { T6-12 } \\
T 6-12 \\
\text { T6-12 } \\
\text { T6-07 } \\
\text { T6-04 } \\
T 6-12\end{array}$ & $\begin{array}{l}x \\
x \\
x \\
x \\
x \\
x \\
x \\
x\end{array}$ & & & \\
\hline$\frac{\text { Lockheed Martin Services, Inc. }}{\text { Central Files }}$ & B1-07 & $x$ & & & \\
\hline$\frac{\text { MACTEC }}{\text { R. D. Potter }}$ & R3-73 & $x$ & & & \\
\hline
\end{tabular}




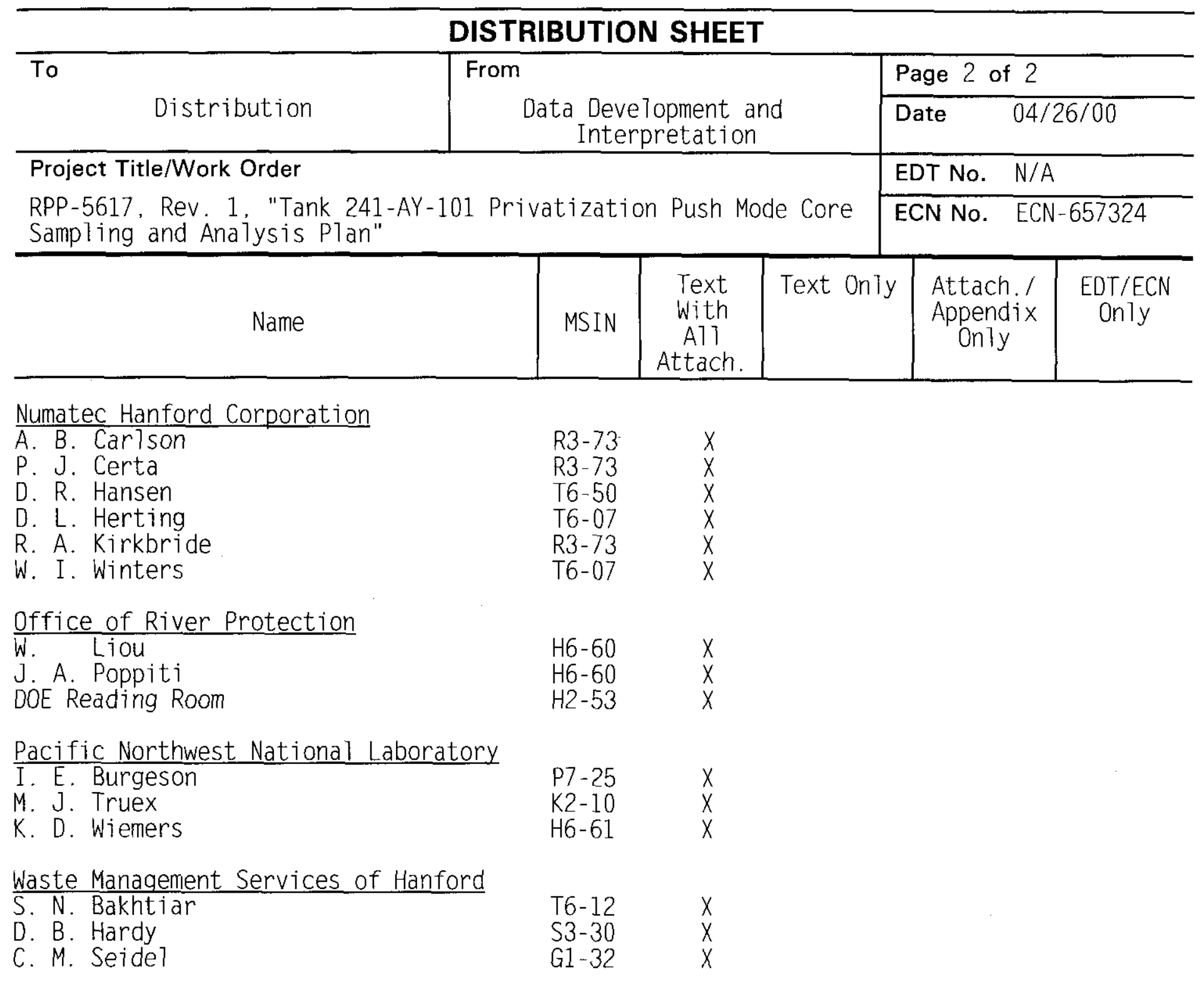

